OPEN ACCESS

Edited by:

Ahmet Eroglu,

Karadeniz Technical University, Turkey

Reviewed by:

Pasquale Sansone,

University of Campania Luigi

Vanvitelli, Italy

Suzanne B. Hanser,

Berklee College of Music,

United States

Anthony Kroyt Brandt,

Rice University, United States

${ }^{*}$ Correspondence:

Luis Ulloa

luis.ulloa@duke.edu

${ }^{\dagger}$ These authors have contributed equally to this work

Specialty section:

This article was submitted to

Intensive Care Medicine and

Anesthesiology,

a section of the journal

Frontiers in Medicine

Received: 01 December 2021

Accepted: 12 January 2022

Published: 04 February 2022

Citation:

Ginsberg JP, Raghunathan K, Bassi G and Ulloa $L$ (2022) Review of

Perioperative Music Medicine: Mechanisms of Pain and Stress

Reduction Around Surgery.

Front. Med. 9:821022.

doi: 10.3389/fmed.2022.821022

\section{Review of Perioperative Music Medicine: Mechanisms of Pain and Stress Reduction Around Surgery}

\author{
J. P. Ginsberg ${ }^{1+}$, Karthik Raghunathan ${ }^{2 \dagger}$, Gabriel Bassi ${ }^{3 \dagger}$ and Luis Ulloa ${ }^{3 * \dagger}$ \\ ${ }^{1}$ Departments of Applied Psychophysiology, Psychology and Statistics, Saybrook University, Pasadena, CA, United States, \\ ${ }^{2}$ Critical Care and Perioperative Population Health Research Unit, Department of Anesthesiology, Duke University Medical \\ Center, Durham, NC, United States, ${ }^{3}$ Department of Anesthesiology, Center for Perioperative Organ Protection, Duke \\ University Medical Center, Durham, NC, United States
}

Clinical-experimental considerations and an approach to understanding the autonomic basis of improved surgical outcomes using Perioperative Music Medicine (PMM) are reviewed. Combined surgical, psycho-physiological, and experimental perspectives on Music Medicine (MM) and its relationship to autonomic nervous system (ANS) function are discussed. Considerations are given to the inter-related perioperative effects of $\mathrm{MM}$ on ANS, pain, and underlying vagal and other neural circuits involved in emotional regulation and dysregulation. Many surgical procedures are associated with significant pain, which is routinely treated with post-operative opioid medications, which cause detrimental side effects and delay recovery. Surgical trauma shifts the sympathetic ANS to a sustained activation impairing physiological homeostasis and causing psychological stress, as well as metabolic and immune dysfunction that contribute to postoperative mortality and morbidity. In this article, we propose a plan to operationalize the study of mechanisms mediating the effects of MM in perioperative settings of orthopedic surgery. These studies will be critical for the implementation of PMM as a routine clinical practice and to determine the potential limitations of MM in specific cohorts of patients and how to improve the treatment.

Keywords: perioperative, music medicine, stress, Heart Rate Variability, autonomic nervous system, vagotomy, review

\section{CLINICAL BENEFITS OF PERIOPERATIVE MUSIC MEDICINE}

Surgery is an "indivisible, indispensable part of health care" around the world $(1,2)$ but it causes significant physiologic stress $(3,4)$. Neural (autonomic) and humoral (circulating or hormonal) mechanisms primarily involve the sympathetic nervous system (SNS) adrenergic and parasympathetic nervous system (PNS) cholinergic branches of the autonomic nervous system (ANS). Current perioperative interventions used to attenuate these stress responses are primarily pharmacologic (anesthetic and analgesic medications) that are associated with a variety of complications (5). The current epidemic of opioid overuse in the United States is fueled in part by excessive perioperative prescriptions around both minor and major surgery $(6,7)$. Non-pharmacologic interventions that complement and integrate with pharmacologic interventions in 
the perioperative setting are thus of critical importance to both decrease psychophysiological stress and decrease opioid use. Perioperative Music Medicine (PMM), defined as listening to pre-recorded music around surgery (8), is an efficacious, safe, and low-cost non-pharmacologic intervention that can be delivered at the point-of-care and reduce opioid use (9-11).

\section{PERIOPERATIVE MUSIC MEDICINE TRANSLATIONAL RESEARCH CONTINUUM}

While the specific mechanisms of PMM remain unclear, preclinical (T0) studies suggest that the auditory pathway must be intact for an effect (Figure 1). In rodent models, physiological effects include a shift from sympathetic toward more parasympathetic autonomic activity, suppression of stress hormones, lowering heart rate, blood pressure, and anxiety, but increasing immune functions (12). In human volunteers (T1 studies) and clinical trial participants (T2 studies), there is a strong body of evidence supporting the efficacy of PMM when compared to ambient noise, ear plugs, or headphones with noise cancellation or white noise (9-11). This translational strategy has been critical in implementing the use of PMM at the Durham VA hospital for the treatment of veterans in North Carolina (Figure 2). In recent meta-analyses of randomized controlled trials (RCTs), PMM was estimated to cause clinically important reduction in pain and anxiety $(\sim 10$ and $20 \mathrm{~mm}$, respectively, on the $100 \mathrm{~mm}$ visual analog scale) (9-11), and decreased opioid use ( $\sim 10 \mathrm{mg}$ lower in morphine equivalents over three postoperative days) (11) (Figure 2). In this section, we briefly review the clinical benefits of PMM on cognitive and affective pathways, as well as the favorable modulation of the ANS and implementation.

\section{PMM Acts on Cognitive and Affective Pathways}

Noise contributes to adverse health outcomes in various settings, and it is a significant stressor in operating rooms (ORs) with an average intensity between 51 to $75 \mathrm{~dB}$ (13). Highest noise levels are found in the ORs in which orthopedic surgery is performed (14). Ear plugs and noise canceling headphones can therefore have benefits, especially around joint replacement surgery (13). Musical activation of the auditory system has been shown to inhibit pain pathways beyond noise cancellation effects (15). Consistent with modern theories of pain (16), PMM has cognitive effects impacting both intensity and quality of pain (9-11), and PMM also lowers anxiety (on average by 5.72 units vs. standard care, when measured by the State Trait Anxiety Index) (17) in both adult and pediatric populations (18). Perioperative Music Medicine decreased pinprick pain, repeated (conditioned) pain stimulus, and also increased the threshold for pressure pain (19). As a distracting stimulus it shifts attention away from competing noxious stimuli (20). Perioperative Music Medicine is especially effective in patients with depression (21), increasing emotional activity centered in the limbic system of the brain which feeds into the common pain pathways (18), produces endogenous opioids (18) and oxytocin $(18,22)$ as well as decreases cortisol (22) and catecholamines $(22,23)$. Thus, PMM may work well in the awake patient. Benefits may be greater when PMM is self-selected (24), because the way in which patients cognitively engage with the music is different when patients choose the type of music.

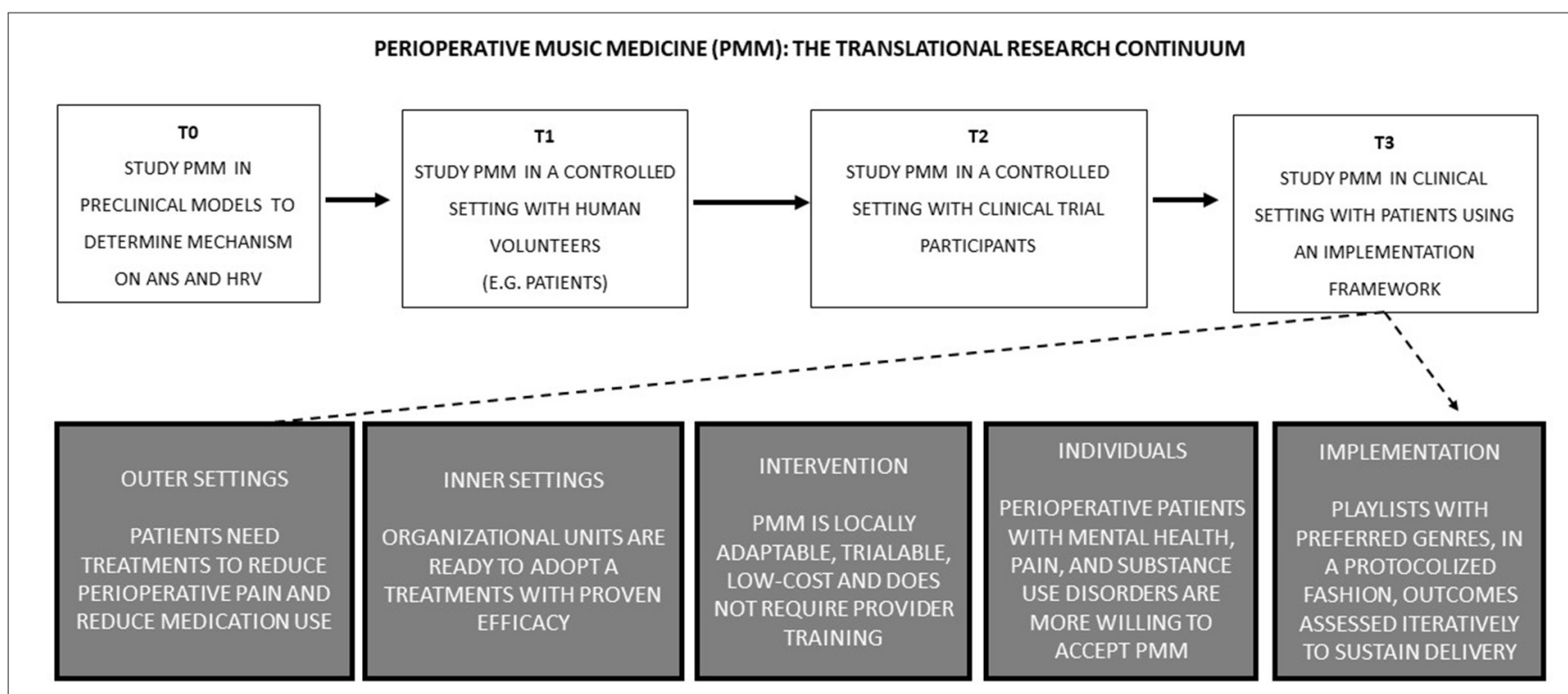

FIGURE 1 | Perioperative Music Medicine (PMM) translational research continuum. Schematic diagram of translational research on PMM for the implementation of new more effective treatments based on mechanistic studies. 


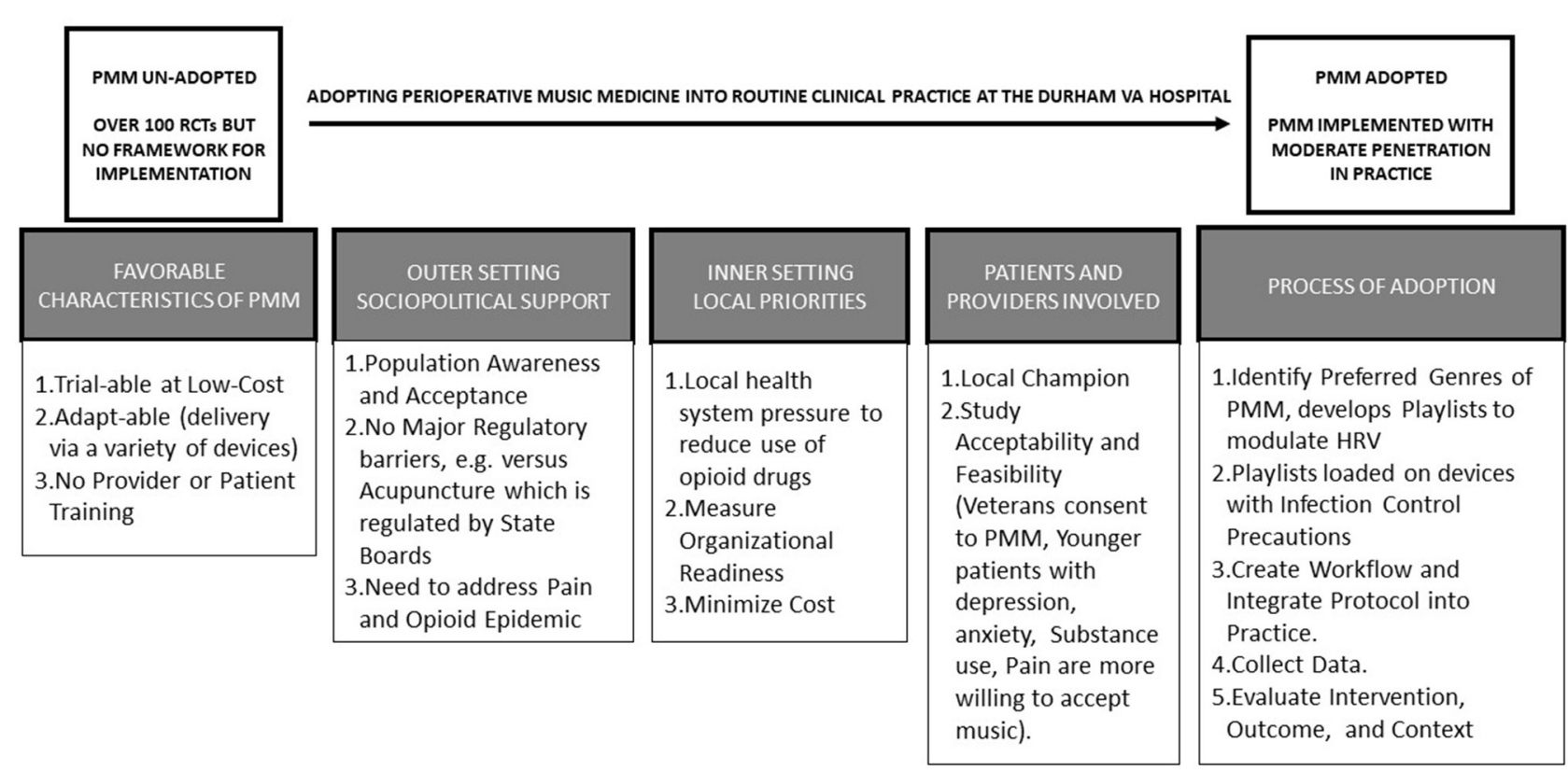

FIGURE 2 | The evidence to practice gap in implementing PMM practice. Schematic representation of the translational strategy from evidence to practice to implement the use PMM into routine clinical practice in orthopedic surgery at the Durham VA Hospital.

\section{PMM Acts on the Autonomic Nervous System}

There is a large body of research on the effects of music on the ANS in healthy subjects, and findings include significant reductions in heart rate (especially when listening to soft music) (25) in both conscious and unconscious volunteers (26). There is now strong evidence that hearing is perceptive during general anesthesia (27), and auditory stimuli-including music under general anesthesia-could modulate the neurohormonal response to surgery. Cortical auditory evoked responses are not abolished by inhalational anesthetics at the concentrations used for surgery (28). This provides a basis to understand the clinical studies where PMM under general anesthesia decreased postoperative pain, analgesic use, anxiety, respiratory rate, blood pressure, cortisol levels, postoperative nausea, and vomiting $(9-11,29)$.

How Heart Rate Variability (HRV) is measured and what it represents is discussed in the next section of this article, but evidence regarding the effects of PMM on HRV is inconsistent (25). While it is well-established that HRV decreases during anesthesia $(30,31)$ and that decreased HRV is associated with greater morbidity and mortality (32), it is not known whether PMM has clinical benefits when changes in HRV are invoked. Since HRV around surgery may aid in evaluation of the perioperative nociception-analgesia balance in both adults and children (32), the effects of PMM may be viewed through the lens of changes in HRV. In other words, the causal pathway from PMM under general or spinal anesthesia to clinical benefits may go through modulation of ANS activity as reflected in changes in the HRV. The last section proposes an experimental setup where HRV is measured throughout the perioperative experience (under general or spinal anesthesia during joint replacement surgery) with subjects randomly assigned to receive or not PMM. We hypothesize that clinical benefits (less pain, anxiety, and medication use) of PMM will depend on its potential to increase the vagal tone and HRV. Our hypothesis is that a decreased HRV could signal a surgery-induced sympathetic stress whereas an increased HRV could signal a parasympathetic resilience to surgical stress. Our hypothesis is that benefits of PMM on surgical outcomes are accompanied by increased HRV, indicating that the effect resides in resilience to surgical trauma due to reduced stress responses.

\section{PMM Can Be Implemented at the Point-of-Care}

For patients to broadly experience the clinical benefits of PMM, it has to be adopted with fidelity and become a part of the local "culture." We recently conducted (T3) research to translate evidence (9-11) into routine delivery of PMM to patients in our daily clinical practice (33). Implementation of PMM was accelerated by the need to respond to the opioid epidemic, and by the relative advantage it offered over other non-pharmacologic interventions including mindfulness, biofeedback, acupuncture, Yoga, and Tai Chi (33). Perioperative Music Medicine can be adapted to the local context and the protocol for PMM can be refined in iterative cycles on a small-scale. Provider credentialing is not required (as in the case of acupuncture) and patient training is also not required (as in biofeedback, Yoga, and Tai Chi). After assessing organizational readiness for implementation and identifying local champions $(34,35)$, the capital investment 
to deploy PMM is modest with the development of playlists based on local patient preferences, purchase of digital music players and covers (for infection control), and extracting data already being collected for clinical purposes (pain scores, medication use, satisfaction scores). Perioperative Music Medicine was welcomed as an exciting complement to opioid and non-opioid analgesia in the perioperative period, and is currently sustained with moderate penetration (such that over two-thirds of the patients who want to receive this intervention actually get it) (33).

Further research is warranted because a path to delivery at the bedside already exists. In the next two sections we review ANS responding to psychophysiological stress and HRV analyses, and the last section proposes combining perioperative clinical with a controlled experimental approach to uncover the mechanisms by which PMM attenuates stress and improves surgical outcomes.

\section{MUSIC MEDICINE AND THE ANS}

Whereas, Music Therapy (MT) is primarily a cognitive rehabilitation method involving a therapist (36-38), Music Medicine (MM) is the delivery of prerecorded music through headphones, musical pillows, or background sound systems as a treatment for clinical disorders, including peri-operative surgical procedures. In our view, music is the active component that accounts for the effectiveness of both approaches, and the two procedures share much in common for disease management. Meta-analyses of RCTs of MM and MT (vs. either no music, white noise, undisturbed bed rest, or usual care in various pain conditions) found that the reduction in pain, anxiety, and opioid use exceeded minimum clinically important differences (39, 40). Surprisingly, and despite the evidence, MM is not widely used. We propose that the central mechanism underlying the benefit of PMM, and MM in general, is the enhancing effect the intervention has on emotional regulation mediated through ANS homeostasis, which can be indexed by HRV. Our overall hypothesis is that MM reduces clinical morbidity by decreasing sympathetic activation and preserving homeostasis of stress metabolism and immune function via strengthening parasympathetic cardiac cholinergic output from the myelinated neurons in the nucleus ambiguous of the vagus nerve. This strengthening of parasympathetic cardiac cholinergic output ("vagal tone") can be indexed quantitatively by changes in HRV.

\section{Autonomic Nervous System Stress and Heart Rate Variability}

Under normal stress, ANS cardiac control reflects an adaptive level of interplay between the PNS and SNS. The PNS produces cardiac deceleration ("rest and digest") via myelinated vagal nerve (CNX) cholinergic output from nucleus ambiguous (also referred to as the "ventral vagal complex") (41) onto the heart. Acetylcholine (ACh) released by ventral vagal stimulation reduces heart rate by activating the M2 muscarinic receptors (M2R) that, in turn, opens the ACh-activated potassium channel to slow the firing of the pacemaker cells in the sinoatrial (SA) node $(42,43)$. The right vagus nerve primarily innervates the SA node and slows its pacemaker whereas the left vagus innervates the AV (atrioventricular) node and slows its conduction of the cardiac impulse to the bundle, but the ventricular myocardium is sparsely innervated by vagal efferent signals (44). The SNS produces cardiac acceleration ("fight or flight") as the individual orients in the social and physical environment (45). Sympathetic efferent nerve endings are present in the SA node and throughout the atria and ventricles (44). In simplified terms, the interplay of the two branches of the ANS allows adaptation to normal stress in the face of everyday challenges by SNS signaling to hypothalamic-pituitary-adrenal (HPA) axis leading to release of corticosteroids (cortisol in humans, corticosterone in rodents) and pro-inflammatory factors from the adrenal gland $(46,47)$. Adrenal glucocorticoid release is regulated by a negative feedback signal to hippocampal glucocorticoid receptors that shuts off stress responding and the corticosteroid and pro-inflammatory neuromodulators when the stress ends (48).

As previously mentioned, the status of dynamic sympathovagal ANS function can be indexed in real time with HRV (49). Heart Rate Variability is basically defined as quantitative analysis of variation in the time interval between heartbeats (interbeat interval, IBI). Heart Rate Variability is an indicator of the magnitude and pattern of changes in inter-beat intervals. The change in successive IBI's (standard deviation of the normal-tonormal pulse, SDNN) in a normal individual is typically in the range of 40-60 ms (50). Thus, HRV can be measured so long as the pulse recording device can sample at a rate $\geq 1,000 \mathrm{~Hz}$ (which corresponds to measurement of a change in IBI of $1 \mathrm{~ms}$ ). A recording of IBI's can be reported with a number of different variables (49). Time domain variables are direct calculations based on IBI's; frequency domain variables are produced by a Fast Fourier Transform of the IBI time series to yield a power spectrum. In general, lower HRV values indicate SNS dominance while higher HRV values indicate a shift to PNS dominance (49).

However, the simple definition of HRV as the pattern of variation between consecutive heartbeats belies the complexity, meaning, and significance of the many different measures of HRV (51) and so there is a potential for apopheniaincorrect conclusions or excessive, unfounded extrapolation (52) - that must be guarded against. Nonetheless, carefully applied quantitative analysis of IBI data can be used to index fundamental systemic clinical physiological processes (53). Because there are numerous methods of analyzing IBI data as various HRV indices [time-domain, frequency-domain, geometric, non-linear, and fractal/complexity $(49,50,54,55)]$, caution must be taken in the selection of the HRV analyses used in any particular context.

Healthy HRV contains a regular pattern of increasing and decreasing IBIs between consecutive beats that increases HRV, while unhealthy HRV is relatively low when little variation between IBIs or random, unorganized differences between consecutive beats exists (50). The rhythm of cardiac acceleration-deceleration is linked to inhalation-exhalation of the respiratory cycle (inhale, cardiac acceleration; exhale, cardiac deceleration), called respiratory sinus arrhythmia (RSA). The regular respiratory cycle-related of increasing and decreasing IBIs approaches a sinusoidal pattern. Slow deep breathing (around six breaths per $\mathrm{min}$ ) is called resonance frequency breathing 
because at that frequency the amplitude of highest to lowest IBI over the respiratory cycle reaches a maximum due to inputs from the baro-receptor and vasomotor reflex maintaining blood pressure homeostasis. Low HRV is the single most accurate clinical predictor of mortality after cardiac events, especially in the elderly (56-59). Current studies reveal that relatively low HRV is also related to several chronic physical illnesses (60), stress (61), and certain forms of mental disorder (62-65), while relatively high HRV is related to optimal physical $(66,67)$, and cognitive (68-70) performance.

\section{Chronic Pain, Chronic Stress, and HRV Are Inter-related}

The sensation of acute nociceptive pain is due to injury to peripheral sub-dermal pain fiber endings, primarily $\mathrm{C}$ and $\mathrm{A} \delta$ fibers (71). C fiber pain transmission is mediated by the ANS (72). When acute nociceptive pain becomes chronic, central pain sensitization often occurs (73). Chronic HPA stress responding due to chronic nociceptive pain interacts with ANS cardiac control and produces persistent sympathetic activation (74). When central pain sensitization occurs, previously sub-threshold synaptic inputs to nociceptive neurons augment action potential output and thus chronic sensitized pain reflects additional contribution from dysregulated CNS and ANS function to the experience of pain (75). However, it is important to note that nociceptive pain that persists after resolution of the initial insult to nociceptive pain fibers cannot be said to be entirely uncoupled from the nociceptive receptors. Sensitized chronic pain states in which tissue inflammation or pathology is not readily apparent (for example, fibromyalgia, irritable bowel syndrome, painful bladder syndrome, or migraine) occur despite the absence of a pathobiological explanation, implicating unresolved nociceptor sensitization in addition to CNS and ANS contributions (76).

Stress from continual pain creates persistent hyperarousal of SNS, known as HPA overdrive (77-79). Hypothalamicpituitary-adrenal overdrive, such as also arises from social and environmental chronic stress, increases glucocorticoid signaling from the adrenal cortex that deregulates the negative feedback signal to shut off stress, down-regulates hippocampal glucocorticoid receptor expression, and ends the normal negative feedback tampering the stress response (80). Chronic sensitized pain is also associated with inhibition of descending cortical pain modulation by periaqueductal gray, rostroventromedial medulla, and serotonergic and adregeneric euromodulators ("nociceptive braking"), producing further proliferation of peripheral proinflammatory cytokines (81). Thus, pain with chronic stress responding becomes a self-reinforcing cycle, causing the pain threshold to be lowered, and a neuro-modulator picture that is equivalent to depression $(77,78,82-84)$. Pain is thus an internal stressor that first triggers a long-term tonic response of the hypothalamic-pituitary adrenal system when pain becomes chronic. Physiological activation leads to both persistent sympathetic arousal lowering HRV (50). Numerous studies have confirmed that HRV variables are lowered by chronic pain (85-94). In addition to chronic stress from chronic pain signals that originate in nociceptive nerve fiber lesions,

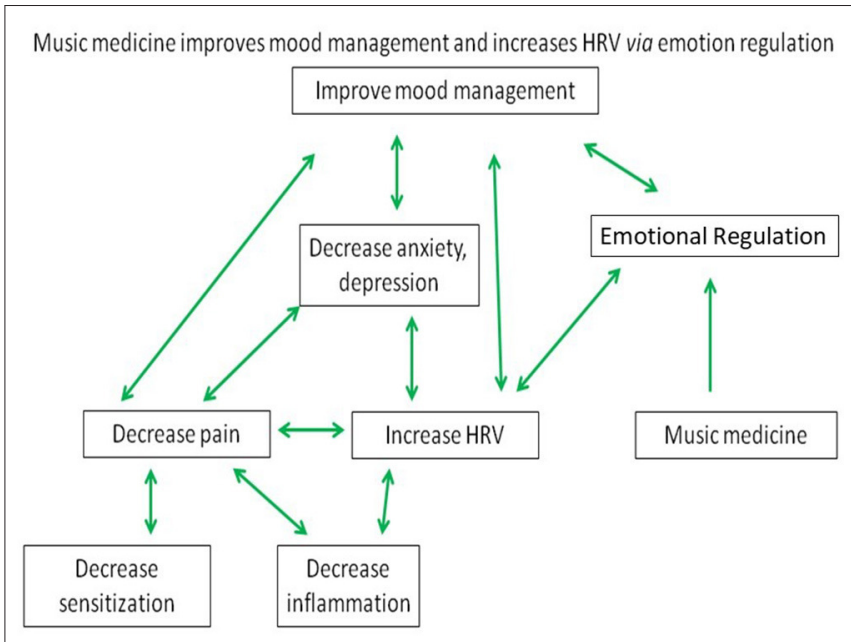

FIGURE 3 | Multifactorial regulation of HRV by music medicine. Proposed Model for Music Medicine to improve mood management and HRV via emotional regulation integrating the regulation of pain, inflammation, anxiety, and depression.

psychological catastrophizing, and unremitting fear rumination further augment the prolonged stress response and are core aspects of chronic pain sensitization $(95,96)$.

\section{HRV, Chronic Pain, and Chronic Stress Are Associated With Inflammation, Depression, and Anxiety}

Inflammation is commonly known to accompany the stress reaction, and the association of HRV with persistent sympathetic arousal typical of stress responding has been well-characterized $(74,77)$. There are several studies showing that HRV and inflammation consistently have an inverse relationship, depicting that inflammation is associated with SNS arousal (97-104).

The observation of decreased HRV associated with depression and anxiety has been widely reported in the literature (105-109) (Figure 3). Findings of increased occurrence of cardio-vascular diseases among patients with Major Depressive Disorder have drawn attention to autonomic regulation of the heart rate as a potential pathophysiological mechanism in depression (110). Dysregulation of autonomic cardiac control resulting in lowered HRV is clearly associated with anxiety and traumatic stress $(111,112)$. State, trait, and clinical expressions of anxiety are considered a restricted response range across biological and behavioral functioning reflecting diminished vagal tone and thereby HRV (62). Thus, chronic pain is readily observable by lowered HRV values because chronic pain, chronic stress, and HRV are inter-related and associated with catecholamines (113), inflammatory mediators (114), depression, and anxiety $(77,115)$.

\section{Many Different Procedures Increase HRV}

Rhythmic stimulation around the $0.1 \mathrm{~Hz}$ frequency from a surprising array of different sources have been shown to have cardiovascular (CV) effects. Rhythmic stimulation that increases HRV includes: instrumental autotraining (116), paced breathing 
(HRVB) (49, 117-120), emotionally salient pictures (121), chanting and toning $(122,123)$, prayer and yoga mantra (124), poetry (125-127), skeletal muscle tension $(128,129)$, orthostatic tilt $(130,131)$, thermal stimulation (132), and neck suction (133). Perhaps the earliest published study of increased HRV due to biofeedback was authored by Vaschillo et al. (116), who observed that Russian cosmonauts could control their breathing while viewing a computer display of their breathing rhythm and heart rate oscillations until their breathing became synchronized with their heart rate oscillations. Although Vaschillo termed this process "instrumental autotraining," the cosmonauts were engaging in a form of biofeedback by controlling their breath cycle to match a computer screen-presented display of heart rate oscillation. Paced slow breathing is very effective at increasing HRV (134-137) and appears to be the most widely used method for increasing heart rate oscillations and associated HRV (138, 139). Current techniques of HRV biofeedback (HRVB) are based on coaching (140) along with the use of a breath pacer (140) in addition to visual display feedback of heart rate oscillation and respiratory cycles.

Increased heart rate oscillations due to paced slow breathing, which is trained in HRVB results from synchronization, or phase-locking, of interacting cycles among physiological systems controlling respiration, heart rate, arterial blood pressure, and vasomotor tone, which increase heart rate oscillations and HRV primarily through the baroreceptor reflex (128, 141-143). As a result, the maximum to minimum IBI during a single breath cycle-a basic definition of HRV (50) - is amplified. Vagal afferent signaling from heart rate to cortex $(141,144-147)$ and a neural pathway based on delta oscillation reduction from prefrontal cortex to vagus (148) influencing vagal efferent signals back to the heart are also recognized.

Increased HRV reflects dampened HPA hyperarousal, calming of the SNS, stimulation of PNS activity, and lowered heart rate, resulting in reduced stress responding. The understanding that heart rate reflects bidirectional heart-brain processes involved in emotional arousal is hardly recent and was put forth by early modern life scientists French physiologist Claude Bernard in 1867 (149) and Charles Darwin in 1872 (150). The relationship of HRV to environmental response and adaptation recently has been re-affirmed (140):

\footnotetext{
“... the complex mix of physiological, behavioral, emotional, and cognitive processes involved in self-regulation and adaptability might have a common basis such that indices of HRV would be associated with all of these various forms of regulation (p. 82). We have proposed that the relationship between HRV and important physiological, cognitive, and emotional regulation functions is due to the ability of HRV to index activity in a flexible network of neural structures that is dynamically organized in response to environmental challenges." (p. 86)
}

Underscoring this relationship between HRV and improved adaptation and stress responding is evidence that a number of psychotherapies that are based on self-regulation of thoughts and emotions that improve well-being-such as Polyvagal-informed Therapy (151, 152), Compassion
Meditation (153), Mindfulness (154-156), Acceptance and Commitment (157), Cognitive Behavior Therapy (158), Forgiveness (159), and Creative Arts (160) - when applied without incorporation of additional training in slow paced breathing, have also been shown to be accompanied by increases in HRV.

\section{Emotional Regulation Is Improved by Increased HRV}

Emotional regulation is the ability to exert control over one's own emotional state, for example controlling thoughts during a challenging situation to reduce anger or anxiety, managing signs of sadness or fear, or focusing on reasons to feel happy or calm. Emotional regulation involves self-monitoring, initiation, maintenance, and modulation of positive and negative emotions, and the avoidance and reduction of high levels of negative affect (161). The function of emotional regulation is adaptation produced by interaction between biological constraints and the physical and social environments. Adaptive emotional regulation is sometimes mistakenly equated with minimization of negative emotion. Healthy emotional regulation is based on flexible but stable behavior patterns of individuals, so both positive and negative emotions are experienced and expressed with a level of intensity that is matched to events, with the goal of achieving successful social interactions and safety.

Perhaps the earliest published study showing that increased HRV due to an intervention using HRVB was beneficial for people with psychological disorder was also authored by Vaschillo et al. (162). HRVB, currently the most widely used technique to increase HRV, is established as a reliable and effective way to improve emotional well-being, mental acuity, and physical function $(105,163)$. HRVB paced breathing is used for clinical intervention of psychological disorder both with and without accompanying "top-down" emotional regulation coaching $(120,164)$. Inducing high amplitude heart rate oscillations via slow paced breathing without intentional emotional regulation nonetheless enhances the emotional regulation neural network function. By manipulating HR oscillations in isolation, Mather (165) demonstrated that an increase in activation occurred in brain regions associated with emotional regulation after heart rate oscillations increased, but activation in the same regions did not occur after oscillations decreased. Furthermore, a behavioral measure of emotional regulation viewing pictures also increased in the high but not in the low HR oscillation group.

\section{MUSIC INCREASES HRV AND IMPROVES EMOTIONAL REGULATION}

We propose that PMM exerts a directional influence that restores autonomic homeostasis that can be indexed by HRV and strengthens emotional regulation, thereby leading to improvements in the individual's state of chronic pain, stress, inflammation, anxiety, and depression. 


\section{Music and HRV}

Beyond the emotional responses, music affects the CV system and influences HRV (26). A systematic review has confirmed that music, as a stimulus acting on the cardiac ANS, increases parasympathetic activity and HRV. However, there is conflicting evidence on whether it is the music itself or listener preferences that matter and the impact of individualized MT of passive listening vs. preferred soundtracks (25). Optimal music for therapy varies between individuals. Self-selected pieces tend to elicit a eustress or joyous response ("positive arousal"), whereas classical music is associated with the highest HRV for parasympathetic dominance (166).

While several studies have established the potential of music to affect HRV as expected, the relationship between music and HRV is complex; for example, exciting music decreases high frequency (HF) HRV power, which is associated with PNS activation, compared to tranquilizing music (167). Overall, excitative music decreases activation of the PNS (168). For example, auditory stimulation with heavy metal music decreased the sympathetic and parasympathetic modulation on the heart, while exposure to a selected classical baroque music reduced only sympathetic regulation on the heart without affecting the parasympathetic activity (169). Acute exposure to heavy metal music also was shown to increase the sympathetic activity in healthy women (170). Likewise, the low frequency (LF) component of HRV and the $\mathrm{LF} / \mathrm{HF}$ ratio increased (indicating increased parasympathetic activation) during sad music.

Autonomic responses to musical stimuli were correlated with subjective preferences regarding the relaxing properties of silence, classical, new age, or romantic melodies (171). The LF/HF ratio was significantly higher when subjects were exposed to "new age" music as compared with silence, while no differences were found with "classical" or "romantic" melodies. These results were related to a reduction in the HF component with "new age" as compared to silence. Subjects' preferences did not correlate with autonomic responses to melodies. The results suggest that "new age" music induced a shift in HRV from higher to lower frequencies, independently of the music preference of the listener.

As a preliminary test of our hypothesis that MM positively affects HRV, we performed a calculation of the effect size of music on HRV specifically in perioperative surgical settings (PMM, presented below).

\section{Music and Emotional Regulation}

The effects of music on emotional regulation have been wellstudied (172) and music has been shown to be an effective strategy to regulate affective states (173). People employ music to induce specific emotional states in everyday situations for the purpose of emotional regulation, usually expressing preference for pieces of music that were emotionally congruent with an emotional situation (174). Musical characteristics such as tempo and rhythm give can change a listener's mood and emotions (175). Desired emotional activation occurs when listening to preferred and familiar music, while undesired activation patterns arise when introducing complexity, heightened, or unfamiliar dissonance emphasizing the connection between music-influenced changes in attention and a link to emotional regulation (176). Brain activity in emotional regulation using music was studied using psychological testing and functional magnetic resonance imaging (fMRI) (177). In this study, neural responses to music were measured in the medial prefrontal cortex (mPFC) in a cohort of 56 participants. Discharge, that is using music to express negative emotions, lowered medial pre-frontal cortex activity in males and diversion, using music to distract from negative emotions, increased medial pre-frontal activity in females. These findings suggest that using music to discharge negative emotions may be associated with a maladaptive pattern of brain function and have long-term negative effects on mental health.

\section{EMOTIONAL REGULATION IS AN IMPORTANT FACTOR IN CHRONIC PAIN, INFLAMMATION, CHRONIC STRESS, AND DEPRESSION AND ANXIETY}

\section{Emotional Regulation and Chronic Pain}

Maladaptive emotional regulation appears to be a risk factor for the development and maintenance of chronic pain, and is associated with psychological co-morbidities of pain (178). Emotional regulation capacities have been empirically linked to variables of pain coping (179), including opioid abuse (180, 181). In general, emotional regulation mediates the relationship between pain and quality of life (182).

\section{Emotional Regulation and Chronic Stress}

Open and RCTs have demonstrated the utility of emotional regulation therapy in treating stress-related conditions (183). Chronic stress is a risk factor for incident CV diseases and emotional regulation moderates the association between chronic stress and CV disease risk. In a large scale $(n=754)$ study, stress, emotional regulation, and CV risk measures were used to test whether emotional regulation mitigates the effect of chronic stress on CV risk (184). Results showed that stress interacted significantly with difficulties in emotional regulation to affect $\mathrm{CV}$ risk. Emotional regulation therapy using a reappraisal strategy alters not only affective components but also brain activity of pathological stress. Reappraisal affects activation of prefrontal cortical areas that reduce activity in limbic areas such as the amygdala, and patients with posttraumatic stress disorder (PTSD) have different brain activity during reappraisal in comparison to individuals without PTSD (185).

\section{Emotional Regulation and Inflammation}

Psychiatric disorders-especially affective disorders including depressive and anxiety disorders are quite common and have been linked to dysfunction in endocrine and immune systems (186). Cytokine signals can access the brain and cause profound changes in neurochemistry, neuroendocrinology, and behavior. For instance, physiological consequences of stressful life experiences derive in part from the effects of stress on the immune response and have relevance for treatment of neuropsychiatric disorders (187). The emotional regulation 
strategies of reappraisal and suppression were studied in relation to inflammation ( $\mathrm{C}$ reactive protein, $\mathrm{CRP}$ ), stress and coronary heart disease in a large cohort of adult offspring from the Collaborative Perinatal Project (188). In this study, the finding emerged that the maladaptive emotional regulation strategy (suppression) was associated with elevated levels of inflammation whereas the adaptive emotional regulation strategy (reappraisal) was associated with lower levels of inflammation. Among couples with marital conflict and poor sleep, people who slept less had higher interleukin-6 (IL-6) and tumor necrosis factor (TNF) production after a marital problem discussion, but adaptive emotional regulation strategies protected who slept less from inflammatory reactivity (189). Furthermore, linkage between autonomic fluctuations measured as HRV synchrony appears to capture engagement with, or an inability to disengage from, a hostile exchange among married couples (190). Stronger HRV synchrony related to situational factors during conflict predicted greater negative affect reactivity, framing conflict as a novel social-biological pathway to inflammation-related diseases.

Emotional regulation and inflammation was modeled as bidirectional pathways linking peripheral inflammation and neural circuitries serving emotional processing and regulation (191). In this model, levels of peripheral inflammation and resting state functional connectivity (rsFC) within the emotional regulation and central executive network are co-regulated. Relationships between inflammation (CRP, IL-6, IL-10, TNF) and rsFC (measured by fMRI) involved in immune-tobrain signaling were found. Key neurobiological correlates of emotional regulation strategies and their effects on mental and physical health include the sub-regions of prefrontal cortex that play a key regulatory role in autonomic, endocrine, and immunological processes. These effects lead to a novel neuroimmuno-affective framework that targets improving emotional regulation, in order to: (1) reduce negative effects associated with depression and/or anxiety; and (2) alter endocrine and immune responses (e.g., reduce inflammation) with changes in activity within (and connectivity between) brain systems that support (successful) emotional regulation (186). Such a framework may be adapted for psychiatric treatment protocols that holistically incorporate neural and immunological biomarkers to promote mental and physical health.

\section{Emotional Regulation and Depression and Anxiety}

For decades, emotional regulation has been thought to be an essential, if underemphasized, feature of mental health (192). Emotional regulation is a factor in mood disorders and emotional dysregulation is prominent in depression and anxiety. People with affective disorders have difficulties implementing the adaptive strategies that are commonly deployed by normal emotional regulation because depression and anxiety associated with various mood and affective disorders negatively impact cognitive processes involved in emotional regulation (193). Depression is understood to be a disorder of emotional regulation (194). In a study of the relationship between depression vulnerability and difficulties with emotional regulation in groups of recovered-depressed and never-depressed participants, emotional suppression was found to be ineffective for down-regulating negative emotions, providing evidence for a role for endogenous emotional regulation but not suppression in depression vulnerability (195). Biological and psychological vulnerabilities associated with anxiety produce increased emotional reactivity, attentional biases toward threat, global tendencies to experience emotions as aversive, and to engage in avoidant processing and behavior (196). In a metaanalysis of Japanese individuals, poor emotional regulation correlated with both depression and anxiety, whereas good emotional regulation had significantly negative correlation with both disorders (197). Meta-analysis of 94 published studies of measures of emotional regulation and well-being revealed relationships in the expected direction (198). These results showed poor emotional regulation strategies had a moderate negative correlation with well-being whereas good emotional regulation strategies had a moderate positive correlation with well-being. Thus, it appears to be important to improve emotional regulation when aiming to improve well-being in people (199) and patients with chronic pain (179, 180), inflammation (200), and stress-related (183), and mental disorders (201-203) (Figure 3).

\section{PUTATIVE PATHWAYS OF EFFECTS OF MM ON CARDIAC AUTONOMIC CONTROL OF HRV, EMOTIONAL REGULATION, AND WELL-BEING}

Physical (such as pain), social, psychological, and environmental stressors cause dysfunctional homeostatic regulation of autonomic cardiac control leading the individual to shift into a state of persistent sympathetic activation and lowered HRV (74). We propose that MM exerts a directional effect on mood and restores autonomic homeostasis via HRV. This shift provides the individual with top-down control of mood management and strengthens emotional regulation, thereby reducing anxiety, depression, inflammation, and sensitized pain. In this article, we seek to better understand MM by considering its mechanistic underpinnings, based on the overarching hypothesis that treatment with MM acts via the vagus nerve to enhance mood, HRV, and emotional self-regulation and reduces sympathetic overactivation, physiologic stress, and inflammation. A better understanding of these mechanisms of MM will facilitate broader application and achievement of greater clinical benefits of MM by seeing how to reduce causative factors leading to mood, pain, and inflammation in clinical settings while at the same time developing, deploying, and improving therapeutic strategies to control autonomic dysfunction.

In our hypothesized model, autonomic control centers in the brainstem bi-directionally influence and are influenced by the cerebral circuits that modulate emotional regulation (depression, anxiety), inflammation, and pain. Cortical higher centers modify the activity of the medullary centers and are particularly important in stimulating CV responses to emotions and stress (44). The literature shows that MM affects HRV in predictable 
ways. It is not surprising that several studies have established that music affects HRV both in healthy individuals (169-171, 204210) and patients (211-224). It is also not surprising that the relationship between music and HRV is a complex one; for example, exciting music has the effect of decreasing HF HRV power, which is associated with PNS activation (49) as compared to tranquilizing music (225).

\section{EFFECT SIZE CALCULATION OF PMM ON HRV}

As a preliminary test of our hypothesis that MM affects $\mathrm{HRV}$ in a clinical (including peri-operative surgical) setting, we performed an effect size calculation. Forty-three published articles were identified as potentially having data that could be used in calculation of effect sizes for effect size of music on HRV in the perioperative surgical settings. Of these, 22 articles reported data that could be used in a calculation of effect size. These studies were selected using two criteria: (1) Independent groups or mixed random assignment design with between-subject data (no non-independent i.e. within-subject data) and (2) tabulated data including group standard deviations (no graphs) were available. All comparisons used a With MM vs. No MM comparison. Comparisons used post-music intervention group means (not pre-post within group changes). Studies were sorted for Independent groups or Mixed Design with random assignment vs. non-independent data and population type. The ratio of LF power to HF power was selected as the variable to be used for determination of effect size because LF/HF ratio is a stable indicator of relative sympathetic to parasympathetic activation under resting conditions and normal respiration. Low frequency is associated with SNS output while HF is associated with PNS output. Thus, a lower LF/HF ratio indicates a relatively higher proportion of PNS output. Six studies using patient samples were included in the effect size analysis. Six published studies met these criteria $(211,212,217,220,221,226)$. Mean LF/HF ratios were: $1.68(0.48)$ for With MM and $2.44(0.65)$ for No MM. Independent sample $t$-test (equal variance shown by Levene Test) was significant $\left[t_{(10)}=-2.29, p\right.$ (one-tailed) $=$ 0.023 ]. The average unweighted effect size estimate was -0.39 (With $\mathrm{MM}<$ No MM).

\section{HYPOTHESIS AND CLINICAL-EXPERIMENTAL RESEARCH}

Our central hypothesis is that activation of efferent myelinated ventral vagal cholinergic output onto the heart is critical for the efficacy of PMM, and MM in general. The resulting strengthening of PNS activity reduces stress responding. The process can be indexed non-invasively by HRV and experimentally demonstrated experimentally by vagotomy.

Surgical trauma induces physiologic stress and dysfunction of the ANS and its modulation of stress and immune responses to surgical trauma $(227,228)$. The mechanism of PMM may be to reduce post-surgical morbidity and improve surgical outcomes by decreasing sustained sympathetic activation and preserving metabolic and immune homeostasis $(26,229)$. We propose to test this hypothesis with a research team that possesses collective clinical and experimental expertise in perioperative settings and experimental animal models. Thus, examining the effects of surgery on autonomic, stress, immune, and mood (depression and anxiety) dysfunction, as well as opioid use and recovery parameters becomes feasible. The long-term goal of this line of research is to maximize clinical benefits of MM by analyzing its mechanism of action. There is a critical need to determine the mechanisms of PMM to maximize its efficacy and broaden the field of application of allied interventions that share the same general mechanism of action that can be deployed into the clinical setting.

\section{DISCUSSION}

The significance of PMM in the surgical contemporary health care system has greater relevance because research on the cognitive and affective pathways of PMM is occurring throughout the Translational Continuum, from preclinical lab studies through implementation in outpatient clinics. Evidence indicates that music affects the ANS, stimulating PNS activity and decreasing postoperative pain and analgesic use even in patients under general anesthesia. Perioperative Music Medicine implemented at the point-of-care is quite feasible and lowcost. However, further research is warranted to elucidate the neural pathways of ANS responding to PMM and whether PMM reduces psychophysiological stress associated with surgery. The model of PMM is supported by empirical data and the effect size calculation of PMM on HRV is significant.

Beyond the surgical suites, MM can be implemented broadly throughout the health care system either by self-administration or with the aid of a music therapist. The impact of chronic pain and chronic stress (due to general stressors in addition to pain) is to lower HRV indices. Chronic pain, chronic stress, and HRV are inter-related and strongly associated with the body's response of inflammation, depression, and anxiety. Heart Rate Variability can be increased and stress responding lowered by a number of interventions other than MM. The common factor underlying the benefit of MM and all the interventions that increase HRV, also variously called "increased resilience" or "well-being," appears to be due to a strengthening of emotional regulation and includes interaction between elements of emotional regulation, chronic pain, chronic stress, inflammation, depression, and anxiety.

A central pathway of the mechanism of ANS response to psychophysiological stress is autonomic control of HRV, reflecting restoration of sympathovagal function from the states of hyper-arousal caused by chronic pain and stress. Vagal afference plays an important role in signaling to cortical and brainstem systems regulating physiological homeostasis. Increasing the level of an individual's HRV stimulates activity in the emotional regulation neural network, while decreased HRV is associated with loss of emotional regulation.

Experimental animal models allow deconstruction of $\mathrm{MM}$ into its component mechanisms. Strategies to understand the 
sensory and physiological underpinnings of MM effects have uncovered the important contributions of music preference and sound perception. Some data have shown that gender plays a role in animal responses to music, and that music may improve task performance in rodents and non-human primates. The brain regions involved in MM responding are being identified. Lowered stress responding due to $\mathrm{MM}$ is indicated by reduced sympathetic and increased parasympathetic activity, and reduced blood pressure and serum corticosterone levels in both normal and hypertensive rodents. Results from studies of a variety of experimentally-induced pathophysiological and disease conditions are consistent with the conclusion that music appears to be an effective treatment.

Surgical procedures are associated with significant pain, which is routinely treated with opioid medications. However, opioids have multiple detrimental side effects, delay recovery, and contribute to complications to postoperative mortality and morbidity. A primary aim of future research will be to conduct a RCT powered based on anticipated effects of PMM on HRV. A related aim of clinical PMM research must be to analyze the associations between HRV and stress and inflammatory markers, and patient-reported outcomes of opioid use, pain, depression, and anxiety.

However, another and equally important aim will be to determine the neuronal networks mediating the mechanism of music modulation of peripheral physiology in experimental animal orthopedic surgery. Functional analyses using pharmacologic, surgical, and genetic approaches to examine how music modifies activity of pathways of neuro-modulation: the HPA and corticosteroids, sympathetic catecholamines, and parasympathetic vagal and cholinergic system in an

\section{REFERENCES}

1. Kim J. Opening Address to the Inaugural "The Lancet Commission on Global Surgery" meeting. The Lancet Commission on Global Surgery. Boston, MA: The World Bank (2014).

2. Meara JG, Leather AJ, Hagander L, Alkire BC, Alonso N, Ameh EA, et al. Global Surgery 2030: evidence and solutions for achieving health, welfare, and economic development. Lancet. (2015) 386:569624. doi: 10.1016/S0140-6736(15)60160-X

3. Gan TJ. Poorly controlled postoperative pain: prevalence, consequences, and prevention. J Pain Res. (2017) 10:2287-98. doi: 10.2147/JPR.S144066

4. Migneault B, Girard F, Albert C, Chouinard P, Boudreault D, Provencher $\mathrm{D}$, et al. The effect of music on the neurohormonal stress response to surgery under general anesthesia. Anesth Analg. (2004) 98:52732. doi: 10.1213/01.ANE.0000096182.70239.23

5. Soffin EM, Lee BH, Kumar KK, Wu CL. The prescription opioid crisis: role of the anaesthesiologist in reducing opioid use and misuse. Br J Anaesth. (2019) 122:e198-208. doi: 10.1016/j.bja.2018.11.019

6. Brummett CM, Waljee JF, Goesling J, Moser S, Lin P, Englesbe MJ, et al. New persistent opioid use after minor and major surgical procedures in US adults. JAMA Surg. (2017) 152:e170504. doi: 10.1001/jamasurg.2017.0504

7. Hah JM, Bateman BT, Ratliff J, Curtin C, Sun E. Chronic opioid use after surgery: implications for perioperative management in the face of the opioid epidemic. Anesth Analg. (2017) 125:1733. doi: 10.1213/ANE.0000000000002458

8. Brandfonbrener AG, Kjelland JM. Music medicine. In: Parncutt R, McPherson G, editors. The Science and Psychology of Music Performance: experimental model of orthopedic surgery. In this protocol, surgical vagotomy and pharmacologic blockade plus genetic knock-out of cholinergic receptors are hypothesized to eliminate the role of the vagus in PMM responding and prevent reduction of inflammation after orthopedic surgery.

The rationale for the need of an animal model in parallel to clinical trials of PMM is compelling. The clinical and animal model aims are interdependent. Surgical patients exposed to PMM may have a range of physiological responses depending on how much they are engaged with the music vs. engaged with everything else going on in the environment. The clinical research model will use HRV and various inflammation biomarkers and psychological instruments to detect how much PMM is working in humans. The HRV response is a way to measure the degree of psychophysiological response. Experimental animal models can mimic surgical trauma and undergo selective neurectomies, genetic, and pharmacological inhibition of the hypothesized neuronal pathways involved in PMM (230, 231). This level of experimental control is only possible in experimental animals. Thus, experimental animal surgery can confirm the hypothesized mechanism of the human model by using specific neural and humoral interruptions. These mechanistic studies will be critical to define the potential limitations of $\mathrm{MM}$ in specific cohorts of patients and how to improve the treatments.

\section{AUTHOR CONTRIBUTIONS}

All authors listed have made a substantial, direct, and intellectual contribution to the work and approved it for publication.
Creative Strategies for Teaching and Learning. Oxford: Oxford University Press (2002) p. 83-96. doi: 10.1093/acprof:oso/9780195138108.003.0006

9. Kühlmann A, De Rooij A, Kroese L, Van Dijk M, Hunink M, Jeekel J. Metaanalysis evaluating music interventions for anxiety and pain in surgery. $\mathrm{Br} \mathrm{J}$ Surg. (2018) 105:773. doi: 10.1002/bjs.10853

10. Hole J, Hirsch M, Ball E, Meads C. Music as an aid for postoperative recovery in adults: a systematic review and meta-analysis. Lancet. (2015) 386:1659-71. doi: 10.1016/S0140-6736(15)60169-6

11. Fu VX, Oomens P, Klimek M, Verhofstad MH, Jeekel J. The effect of perioperative music on medication requirement and hospital length of stay: a meta-analysis. Ann Surg. (2020) 272:961. doi: 10.1097/SLA.0000000000003506

12. Kühlmann AYR, de Rooij A, Hunink MGM, De Zeeuw CI, Jeekel J. Music affects rodents: a systematic review of experimental research. Front Behav Neurosci. (2018). 12:301. doi: 10.3389/fnbeh.2018.00301

13. Hasfeldt D, Laerkner E, Birkelund R. Noise in the operating room-what do we know? A review of the literature. J PeriAnesth Nurs. (2010) 25:3806. doi: 10.1016/j.jopan.2010.10.001

14. Siegel MG. The risk of noise-induced hearing loss performing knee replacement surgery. Noise Health. (2019) 21:1838. doi: 10.4103/nah.NAH_22_19

15. Koch ME, Kain ZN, Ayoub C, Rosenbaum SH. The sedative and analgesic sparing effect of music. Anesthesiology. (1998) 89:300-6. doi: 10.1097/00000542-199808000-00005

16. Melzack R, Casey KL. Sensory, motivational, and central control determinants of pain: a new conceptual model. In: Kenshalo DR, editor. The Skin Senses. Vol. 1. Springfield, IL: Charles C Thomas (1968) p. 423-39. 
17. Bradt J, Dileo C, Shim M. Music interventions for preoperative anxiety. Cochrane Database Syst Rev. (2013) 2013:CD006908. doi: 10.1002/14651858.CD006908.pub2

18. Bernatzky G, Presch M, Anderson M, Panksepp J. Emotional foundations of music as a non-pharmacological pain management tool in modern medicine. Neurosci Biobehav Rev. (2011) 35:1989-99. doi: 10.1016/j.neubiorev.2011.06.005

19. Chai PR, Gale JY, Patton ME, Schwartz E, Jambaulikar GD, Wade Taylor S, et al. The impact of music on nociceptive processing. Pain Med. (2020) 21:3047-54. doi: 10.1093/pm/pnaa070

20. Howlin C, Rooney B. The cognitive mechanisms in music listening interventions for pain: a scoping review. J Music Ther. (2020) 57:12767. doi: $10.1093 / \mathrm{jmt} /$ thaa003

21. Aalbers S, Fusar-Poli L, Freeman RE, Spreen M, Ket JC, Vink AC, et al. Music therapy for depression. Cochrane Database Syst Rev. (2017) 2017:CD004517. doi: 10.1002/14651858.CD004517.pub3

22. Fu VX, Oomens P, Sneiders D, van den Berg SAA, Feelders RA, Wijnhoven $\mathrm{BPL}$, et al. The effect of perioperative music on the stress response to surgery: a meta-analysis. J Surg Res. (2019) 244:444-55. doi: 10.1016/j.jss.2019.06.052

23. Wu PY, Huang ML, Lee WP, Wang C, Shih WM. Effects of music listening on anxiety and physiological responses in patients undergoing awake craniotomy. Complement Ther Med. (2017) 32:56-60. doi: 10.1016/j.ctim.2017.03.007

24. Engwall M, Duppils GS. Music as a nursing intervention for postoperative pain: a systematic review. J Perianesth Nurs. (2009) 24:370-83. doi: 10.1016/j.jopan.2009.10.013

25. Mojtabavi H, Saghazadeh A, Valenti VE, Rezaei N. Can music influence cardiac autonomic system? A systematic review and narrative synthesis to evaluate its impact on heart rate variability. Complement Ther Clin Pract. (2020) 39:101162. doi: 10.1016/j.ctcp.2020.101162

26. Ellis RJ, Thayer JF. Music and autonomic nervous system (dys) function. Music Percept. (2010) 27:317-26. doi: 10.1525/mp.2010.27.4.317

27. Nowak H, Zech N, Asmussen S, Rahmel T, Tryba M, Oprea G, et al. Effect of therapeutic suggestions during general anaesthesia on postoperative pain and opioid use: multicentre randomised controlled trial. BMJ. (2020) 371:m4284. doi: 10.1136/bmj.m4284

28. Thornton C, Catley DM, Jordan C, Lehane JR, Royston D, Jones JG. Enflurane anaesthesia causes graded changes in the brainstem and early cortical auditory evoked response in man. Br J Anaesth. (1983) 55:47986. doi: $10.1093 / \mathrm{bja} / 55.6 .479$

29. Fu VX, Sleurink KJ, Janssen JC, Wijnhoven BPL, Jeekel J, Klimek M. Perception of auditory stimuli during general anesthesia and its effects on patient outcomes: a systematic review and meta-analysis. Can J Anaesth. (2021) 68:1231-53. doi: 10.1007/s12630-021-02015-0

30. Kato M, Komatsu T, Kimura T, Sugiyama F, Nakashima K, Shimada Y. Spectral analysis of heart rate variability during isoflurane anesthesia. Anesthesiology. (1992) 77:669-74. doi: 10.1097/00000542-199210000-00009

31. Mäenpää $M$, Penttilä $J$, Laitio $T$, Kaisti K, Kuusela T, Hinkka S, et al. The effects of surgical levels of sevoflurane and propofol anaesthesia on heart rate variability. Eur J Anaesthesiol. (2007) 24:626-33. doi: 10.1017/S0265021507000129

32. Anderson TA. Heart rate variability: implications for perioperative anesthesia care. Curr Opin Anesthesiol. (2017) 30:6917. doi: 10.1097/ACO.0000000000000530

33. Carter JE, Pyati S, Kanach FA, Maxwell AMW, Belden CM, Shea CM, et al. Implementation of perioperative music using the consolidated framework for implementation research. Anesth Analg. (2018) 127:623-31. doi: 10.1213/ANE.00000000000 03565

34. Polascik BA, Tan DJA, Raghunathan K, Kee HM, Lee A, Sng BL, et al. Acceptability and feasibility of perioperative music listening: a rapid qualitative inquiry approach. J Music Ther. (2020) 58:4369. doi: $10.1093 / \mathrm{jmt} /$ thaa014

35. Tan DJA, Polascik BA, Kee HM, Hui Lee AC, Sultana R, Kwan $\mathrm{M}$, et al. The effect of perioperative music listening on patient satisfaction, anxiety, and depression: a quasiexperimental study. Anesthesiol Res Pract. (2020) 2020:3761398. doi: 10.1155/2020/37 61398
36. Gold C, Erkkilä J, Bonde LO, Trondalen G, Maratos A, Crawford MJ. Music therapy or music medicine? Psychother Psychosom. (2011) 80:304. doi: 10.1159/000323166

37. Dileo C. Music Therapy and Medicine: Theoretical and Clinical Applications. Silver Spring, MD: American Music Therapy Association (1999).

38. Dileo C, Bradt J. Medical Music Therapy: A Meta-Analysis and Agenda for Future Research. Cherry Hill, NJ: Jeffrey Books (2005).

39. Cepeda MS, Carr DB, Lau J, Alvarez H. Music for pain relief. Cochrane Database Syst Rev. (2006) 2006:CD004843. doi: 10.1002/14651858.CD004843.pub2

40. Cole LC, LoBiondo-Wood G. Music as an adjuvant therapy in control of pain and symptoms in hospitalized adults: a systematic review. Pain Manag Nurs. (2014) 15:406-25. doi: 10.1016/j.pmn.2012.08.010

41. Porges S. The Polyvagal Theory: Neurophysiological Foundations of Emotions, Attachment, Communication, and Self-Regulation. New York, NY: WW Norton and Company. (2011).

42. Moss R, Sachse FB, Moreno-Galindo EG, Navarro-Polanco RA, TristaniFirouzi M, Seemann G. Modeling effects of voltage dependent properties of the cardiac muscarinic receptor on human sinus node function. PLoS Comput Biol. (2018) 14:e1006438. doi: 10.1371/journal.pcbi.1006438

43. Sakmann B, Noma A, Trautwein W. Acetylcholine activation of single muscarinic $\mathrm{K}+$ channels in isolated pacemaker cells of the mammalian heart. Nature. (1983) 303:250-3. doi: 10.1038/303250a0

44. Klabunde R. Cardiovascular Physiology Concepts. 3rd ed. Alphen aan den Rijn: Wolters Kluwer. (2021).

45. Porges S. Orienting in a defensive world: mammalian modification of our evolutionary heritage. A polyvagal theory. Psychophysiology. (1995) 32:30118. doi: 10.1111/j.1469-8986.1995.tb01213.x

46. Boscolo P, Youinou P, Theoharides TC, Cerulli G, Conti P. Environmental and occupational stress and autoimmunity. Autoimmun Rev. (2008) 7:3403. doi: 10.1016/j.autrev.2007.12.003

47. Herman JP, McKlveen JM, Ghosal S, Kopp B, Wulsin A, Makinson R, et al. Regulation of the hypothalamic-pituitary-adrenocortical stress response. Compr Physiol. (2016) 6:603-21. doi: 10.1002/cphy.c150015

48. Gjerstad JK, Lightman SL, Spiga F. Role of glucocorticoid negative feedback in the regulation of HPA axis pulsatility. Stress. (2018) 21:40316. doi: $10.1080 / 10253890.2018 .1470238$

49. Camm A, Malik M, Bigger J, Breithardt G, Cerutti S, Cohen R, et al. Heart rate variability: standards of measurement, physiological interpretation and clinical use. Task Force of the European Society of Cardiology and the North American Society of Pacing and Electrophysiology. Circulation. (1996) 93:1043-65.

50. Shaffer F, Ginsberg JP. An overview of heart rate variability metrics and norms. Front Publ Health. (2017) 5:258. doi: 10.3389/fpubh.2017.00258

51. Bourdin A, Bjermer L, Brightling C, Brusselle GG, Chanez P, Chung $\mathrm{KF}$, et al. ERS/EAACI statement on severe exacerbations in asthma in adults: facts, priorities and key research questions. Eur Respir J. (2019). 54:1900900. doi: 10.1183/13993003.00900-2019

52. Gibson W. Pattern Recognition. New York, NY: Penguin Random House LLC. (2003).

53. Lombardi F. Clinical implications of present physiological understanding of HRV components. Card Electrophysiol Rev. (2002) 6:245-9. doi: 10.1023/a:1016329008921

54. Goldberger AL, Amaral LA, Hausdorff JM. Ivanov PCh, Peng CK, Stanley HE. Fractal dynamics in physiology: alterations with disease and aging. Proc Natl Acad Sci USA. (2002) 99(Suppl 1):2466-72. doi: 10.1073/pnas.012579499

55. Gronwald T, Rogers B, Hoos O. Fractal correlation properties of heart rate variability: a new biomarker for intensity distribution in endurance exercise and training prescription? Front Physiol. (2020) 11:550572. doi: 10.3389/fphys.2020.550572

56. Kleiger RE, Miller JP, Bigger JT, Moss AJ. Decreased heart rate variability and its association with increased mortality after acute myocardial infarction. $A m$ J Cardiol. (1987) 59:256-62. doi: 10.1016/0002-9149(87)90795-8

57. Tsuji H, Larson MG, Venditti FJ, Manders ES, Evans JC, Feldman $\mathrm{CL}$, et al. Impact of reduced heart rate variability on risk for cardiac events. The Framingham Heart Study. Circulation. (1996) 94:28505. doi: 10.1161/01.CIR.94.11.2850 
58. Buccelletti E, Gilardi E, Scaini E, Galiuto L, Persiani R, Biondi A, et al. Heart rate variability and myocardial infarction: Systematic literature review and metanalysis. Eur Rev Med Pharmacol Sci. (2009) 13:299-307. Available online at: https://www.europeanreview.org/wp/wp-content/uploads/650.pdf

59. Tsuji H, Venditti FJ, Manders ES, Evans JC, Larson MG, Feldman $\mathrm{CL}$, et al. Reduced heart rate variability and mortality risk in an elderly cohort. The Framingham Heart Study. Circulation. (1994) 90:87883. doi: 10.1161/01.CIR.90.2.878

60. Bassi GS, Kanashiro A, Rodrigues GJ, Cunha FQ, Coimbra NC, Ulloa L. Brain stimulation differentially modulates nociception and inflammation in aversive and non-aversive behavioral conditions. Neuroscience. (2018) 383:191-204. doi: 10.1016/j.neuroscience.2018.05.008

61. Kim HG, Cheon EJ, Bai DS, Lee YH, Koo BH. Stress and heart rate variability: a meta-analysis and review of the literature. Psychiatry Investig. (2018) 15:235-45. doi: 10.30773/pi.2017.08.17

62. Friedman BH. An autonomic flexibility-neurovisceral integration model of anxiety and cardiac vagal tone. Biol Psychol. (2007) 74:185-99. doi: 10.1016/j.biopsycho.2005.08.009

63. Koch C, Wilhelm M, Salzmann S, Rief W, Euteneuer F. A meta-analysis of heart rate variability in major depression. Psychol Med. (2019) 49:194857. doi: 10.1017/S0033291719001351

64. Meeus M, Goubert D, De Backer F, Struyf F, Hermans L, Coppieters I, et al. Heart rate variability in patients with fibromyalgia and patients with chronic fatigue syndrome: a systematic review. Semin Arthritis Rheum. (2013) 43:279-87. doi: 10.1016/j.semarthrit.2013.03.004

65. Sack M, Hopper JW, Lamprecht F. Low respiratory sinus arrhythmia and prolonged psychophysiological arousal in posttraumatic stress disorder: heart rate dynamics and individual differences in arousal regulation. Biol Psychiatry. (2004) 55:284-90. doi: 10.1016/S0006-3223(03)00677-2

66. Makivić B, Nikić Djordjević M, Willis MS. Heart Rate Variability (HRV) as a tool for diagnostic and monitoring performance in sport and physical activities. J Exerc Physiol. (2013). 16:103-31. Available online at: https://www.researchgate.net/profile/Bojan_Makivic/publication/ 285968540_Heart_Rate_Variability_HRV_as_a_Tool_for_Diagnostic_ and_Monitoring_Performance_in_Sport_and_Physical_Activities/links/ 566f019e08ae52dd6c12d205.pdf

67. Plews DJ, Laursen PB, Stanley J, Kilding AE, Buchheit M. Training adaptation and heart rate variability in elite endurance athletes: opening the door to effective monitoring. Sports Med. (2013) 43:773-81. doi: 10.1007/s40279-013-0071-8

68. Hansen AL, Johnsen BH, Thayer JF. Vagal influence on working memory and attention. Int J Psychophysiol. (2003) 48:263-74. doi: 10.1016/S0167-8760(03)00073-4

69. Siennicka A, Quintana DS, Fedurek P, Wijata A, Paleczny B, Ponikowska B, et al. Resting heart rate variability, attention and attention maintenance in young adults. Int J Psychophysiol. (2019) 143:126-31. doi: 10.1016/j.ijpsycho.2019.06.017

70. Thayer JF, Hansen AL, Saus-Rose E, Johnsen BH. Heart rate variability, prefrontal neural function, and cognitive performance: the neurovisceral integration perspective on self-regulation, adaptation, and health. Ann Behav Med. (2009) 37:141-53. doi: 10.1007/s12160-009-9101-z

71. Dubin AE, Patapoutian A. Nociceptors: the sensors of the pain pathway. $J$ Clin Invest. (2010) 120:3760-72. doi: 10.1172/JCI42843

72. Cortelli P, Giannini G, Favoni V, Cevoli S, Pierangeli G. Nociception and autonomic nervous system. Neurol Sci. (2013) 34(Suppl 1):S416. doi: 10.1007/s10072-013-1391-z

73. Woolf CJ. Central sensitization: implications for the diagnosis and treatment of pain. Pain. (2011). 152(3 Suppl):S2-15. doi: 10.1016/j.pain.2010.09.030

74. Gharbo RS. Autonomic rehabilitation: adapting to change. Phys Med Rehabil Clin N Am. (2020) 31:633-48. doi: 10.1016/j.pmr.2020.07.003

75. Latremoliere A, Woolf CJ. Central sensitization: a generator of pain hypersensitivity by central neural plasticity. J Pain. (2009) 10:895926. doi: 10.1016/j.jpain.2009.06.012

76. Gold MS, Gebhart GF. Nociceptor sensitization in pain pathogenesis. Nat Med. (2010) 16:1248-57. doi: 10.1038/nm.2235

77. Blackburn-Munro G, Blackburn-Munro R. Chronic pain, chronic stress and depression: coincidence or consequence? J Neuroendocrinol. (2001) 13:100923. doi: 10.1046/j.0007-1331.2001.00727.x
78. Blackburn-Munro G, Blackburn-Munro R. Pain in the brain: are hormones to blame? Trends Endocrinol Metab. (2003) 14:20-7. doi: 10.1016/S1043-2760(02)00004-8

79. Levy BH, Tasker JG. Synaptic regulation of the hypothalamic-pituitaryadrenal axis and its modulation by glucocorticoids and stress. Front Cell Neurosci. (2012) 6:24. doi: 10.3389/fncel.2012.00024

80. Turecki G, Meaney MJ. Effects of the social environment and stress on glucocorticoid receptor gene methylation: a systematic review. Biol Psychiatry. (2016) 79:87-96. doi: 10.1016/j.biopsych.2014.11.022

81. Ossipov MH, Morimura K, Porreca F. Descending pain modulation and chronification of pain. Curr Opin Support Palliat Care. (2014) 8:14351. doi: 10.1097/SPC.0000000000000055

82. Nestler EJ, Barrot M, DiLeone RJ, Eisch AJ, Gold SJ, Monteggia LM. Neurobiology of depression. Neuron. (2002) 34:13-25. doi: 10.1016/S0896-6273(02)00653-0

83. Palazidou E. The neurobiology of depression. Br Med Bull. (2012) 101:12745. doi: 10.1093/bmb/lds004

84. Robinson MJ, Edwards SE, Iyengar S, Bymaster F, Clark M, Katon W. Depression and pain. Front Biosci. (2009) 14:5031-51. doi: 10.2741/3585

85. Appelhans B, Luecken L. Heart rate variability as an index of regulated emotional responding. Rev Gen Psychol. (2006) 10:229-40. doi: 10.1037/1089-2680.10.3.229

86. Berry ME, Chapple IT, Ginsberg JP, Gleichauf KJ, Meyer JA, Nagpal ML. Non-pharmacological intervention for chronic pain in veterans: a pilot study of heart rate variability biofeedback. Glob Adv Health Med. (2014) 3:28-33. doi: 10.7453/gahmj.2013.075

87. Burch JB, Ginsberg JP, McLain AC, Franco R, Stokes S, Susko K, et al. Symptom management among cancer survivors: randomized pilot intervention trial of heart rate variability biofeedback. Appl Psychophysiol Biofeedback. (2020) 45:99-108. doi: 10.1007/s10484-020-09462-3

88. Gharbo R, Bagherpour R, Lazarus N, Mehrdady R, Ginsberg JP, Burch $\mathrm{JB}$, et al. Untangling chronic pain and hyperarousal with heart rate variability: a case report symptom management among cancer survivors: randomized pilot intervention trial of heart rate variability biofeedback. Practical Pain Manage. (2019) 19:33-6. Available online at: https://www. practicalpainmanagement.com/treatments/interventional/untanglingchronic-pain-hyperarousal-heartrate-variability-case-report

89. Hallman DM, Olsson EM, von Scheele B, Melin L, Lyskov E. Effects of heart rate variability biofeedback in subjects with stress-related chronic neck pain: a pilot study. Appl Psychophysiol Biofeedback. (2011) 36:7180. doi: 10.1007/s10484-011-9147-0

90. Hassett AL, Radvanski DC, Vaschillo EG, Vaschillo B, Sigal LH, Karavidas $\mathrm{MK}$, et al. A pilot study of the efficacy of heart rate variability (HRV) biofeedback in patients with fibromyalgia. Appl Psychophysiol Biofeedback. (2007) 32:1-10. doi: 10.1007/s10484-006-9028-0

91. Kaiser RS, Mooreville M, Kannan K. Psychological interventions for the management of chronic pain: a review of current evidence. Curr Pain Headache Rep. (2015) 19:43. doi: 10.1007/s11916-015-0517-9

92. Kolacz J, Porges SW. Chronic diffuse pain and functional gastrointestinal disorders after traumatic stress: pathophysiology through a polyvagal perspective. Front Med. (2018) 5:145. doi: 10.3389/fmed.2018.00145

93. Provan SA, Olstad DS, Solberg EE, Smedslund G, Dagfinrud H. Evidence of reduced parasympathetic autonomic regulation in inflammatory joint disease: a meta-analyses study. Semin Arthritis Rheum. (2018) 48:13440. doi: 10.1016/j.semarthrit.2017.11.010

94. Sherman RA. Pain Assessment and Intervention from a Psychophysiological Perspective. Wheat Ridge, CO: Association for Applied Psychophysiology and Biofeedback (AAPB). (2004).

95. Quartana PJ, Campbell CM, Edwards RR. Pain catastrophizing: a critical review. Expert Rev Neurother. (2009) 9:745-58. doi: 10.1586/ern.09.34

96. Seminowicz DA, Davis KD. Cortical responses to pain in healthy individuals depends on pain catastrophizing. Pain. (2006) 120:297306. doi: 10.1016/j.pain.2005.11.008

97. Ackland GL, Minto G, Clark M, Whittle J, Stephens RCM, Owen $\mathrm{T}$, et al. Autonomic regulation of systemic inflammation in humans: a multi-center, blinded observational cohort study. Brain Behav Immun. (2018) 67:47-53. doi: 10.1016/j.bbi.2017. 08.010 
98. Adlan AM. Veldhuijzen van Zanten JJCS, Lip GYH, Paton JFR, Kitas GD, Fisher JP. Cardiovascular autonomic regulation, inflammation and pain in rheumatoid arthritis. Auton Neurosci. (2017) 208:13745. doi: 10.1016/j.autneu.2017.09.003

99. Aeschbacher S, Schoen T, Dorig L, Kreuzmann R, Neuhauser C. SchmidtTrucksäss A, et al. Heart rate, heart rate variability and inflammatory biomarkers among young and healthy adults. Ann Med. (2017) 49:1923. doi: 10.1080/07853890.2016.1226512

100. Bell KA, Kobayashi I, Chen Y, Mellman TA. Nocturnal autonomic nervous system activity and morning proinflammatory cytokines in young adult African Americans. J Sleep Res. (2017) 26:510-5. doi: 10.1111/jsr.12480

101. Hasty F, García G, Dávila CH, Wittels SH, Hendricks S, Chong S. Heart rate variability as a possible predictive marker for acute inflammatory response in COVID-19 patients. Mil Med. (2020) 186:e348. doi: 10.1093/milmed/usaa405

102. Jarczok MN, Kleber ME, Koenig J, Loerbroks A, Herr RM, Hoffmann K, et al. Investigating the associations of self-rated health: heart rate variability is more strongly associated than inflammatory and other frequently used biomarkers in a cross sectional occupational sample. PLoS ONE. (2015) 10:e0117196. doi: 10.1371/journal.pone.0117196

103. Weber CS, Thayer JF, Rudat M, Wirtz PH, Zimmermann-Viehoff F, Thomas A, et al. Low vagal tone is associated with impaired post stress recovery of cardiovascular, endocrine, and immune markers. Eur J Appl Physiol. (2010) 109:201-11. doi: 10.1007/s00421-009-1341-x

104. Lampert R, Bremner JD, Su S, Miller A, Lee F, Cheema F, et al. Decreased heart rate variability is associated with higher levels of inflammation in middle-aged men. Am Heart J. (2008). 156:759.e17. doi: 10.1016/j.ahj.2008.07.009

105. Lehrer P, Kaur K, Sharma A, Shah K, Huseby R, Bhavsar J, et al. Heart rate variability biofeedback improves emotional and physical health and performance: a systematic review and meta analysis. Appl Psychophysiol Biofeedback. (2020) 45:109-29. doi: 10.1007/s10484-020-09466-Z

106. Hage B, Sinacore J, Heilman K, Porges SW, Halaris A. Heart rate variability predicts treatment outcome in major depression. J Psychiatry Brain Sci. (2017). 2:1. doi: 10.20900/jpbs.20170017

107. Rottenberg J, Chambers AS, Allen JJ, Manber R. Cardiac vagal control in the severity and course of depression: the importance of symptomatic heterogeneity. J Affect Disord. (2007) 103:173-9. doi: 10.1016/j.jad.2007.01.028

108. Kemp AH, Quintana DS, Gray MA, Felmingham KL, Brown K, Gatt JM. Impact of depression and antidepressant treatment on heart rate variability: a review and meta-analysis. Biol Psychiatry. (2010) 67:106774. doi: 10.1016/j.biopsych.2009.12.012

109. Kanashiro A, Franchin M, Bassi GS, Reis Santana DA, Cunha TM, Cunha FQ, et al. Inhibition of spinal p38 MAPK prevents articular neutrophil infiltration in experimental arthritis via sympathetic activation. Fundam Clin Pharmacol. (2018) 32:155-62. doi: 10.1111/fcp.12338

110. Hare DL, Toukhsati SR, Johansson P, Jaarsma T. Depression and cardiovascular disease: a clinical review. Eur Heart J. (2014) 35:136572. doi: 10.1093/eurheartj/eht462

111. Ginsberg J. Editorial: Dysregulation of autonomic cardiac control by traumatic stress and anxiety. Front Psychol. (2016) 7:945. doi: 10.3389/fpsyg.2016.00945

112. Schneider M, Schwerdtfeger A. Autonomic dysfunction in posttraumatic stress disorder indexed by heart rate variability: a meta-analysis. Psychol Med. (2020) 50:1937-48. doi: 10.1017/S003329172000207X

113. Sadhasivam S, Chidambaran V. Pharmacogenomics of opioids and perioperative pain management. Pharmacogenomics. (2012) 13:171940. doi: $10.2217 /$ pgs. 12.152

114. Zhu Y, Wang S, Wu H, Wu Y. Effect of perioperative parecoxib on postoperative pain and local inflammation factors PGE2 and IL-6 for total knee arthroplasty: a randomized, double-blind, placebo-controlled study. Eur J Orthop Surg Traumatol. (2014) 24:395-401. doi: 10.1007/s00590-013-1203-4

115. Sheng J, Liu S, Wang Y, Cui R, Zhang X. The link between depression and chronic pain: neural mechanisms in the brain. Neural Plast. (2017) 2017:9724371. doi: 10.1155/2017/9724371
116. Vaschillo EG, Zingerman AM, Konstantinov MA, Menitsky DN. Research of the resonance characteristics for the cardiovascular system. Hum Physiol. (1983). 9:257-65.

117. Vaschillo B, Lehrer PM. Characteristics of resonance in heart rate variability stimulated by biofeedback. Appl Psychophysiol Biofeedback. (2006) 31:12942. doi: 10.1007/s10484-006-9009-3

118. Song HS, Lehrer PM. The effects of specific respiratory rates on heart rate and heart rate variability. Appl Psychophysiol Biofeedback. (2003) 28:1323. doi: 10.1023/a:1022312815649

119. Lehrer PM, Vaschillo E, Vaschillo B. Resonant frequency biofeedback training to increase cardiac variability: rationale and manual for training. Appl Psychophysiol Biofeedback. (2000) 25:177-91. doi: 10.1023/A:1009554825745

120. Lehrer P, Vaschillo B, Zucker T, Graves J, Katsamanis M, Aviles M, et al. Protocol for heart rate variability biofeedback training. Biofeedback (Online). (2013) 41:98. doi: 10.5298/1081-5937-41.3.08

121. Vaschillo EG, Bates ME, Vaschillo B, Lehrer P, Udo T, Mun EY, et al. Heart rate variability response to alcohol, placebo, and emotional picture cue challenges: effects of 01-Hz stimulation. Psychophysiology. (2008) 45:84758. doi: 10.1111/j.1469-8986.2008.00673.x

122. Jovanov E, editor. Real-time monitoring of spontaneous resonance in heart rate variability. 2008 30th Annual International Conference of the IEEE Engineering in Medicine and Biology Society. IEEE. Vancouver, BC (2008). doi: 10.1109/IEMBS.2008.4649781

123. Bernardi NF, Snow S, Peretz I, Perez HO, Sabet-Kassouf N, Lehmann A. Cardiorespiratory optimization during improvised singing and toning. Sci Rep. (2017) 7:1-8. doi: 10.1038/s41598-017-07171-2

124. Bernardi L, Sleight P, Bandinelli G, Cencetti S, Fattorini L, WdowczycSzulc J, et al. Effect of rosary prayer and yoga mantras on autonomic cardiovascular rhythms: comparative study. BMJ. (2001) 323:1446-9. doi: 10.1136/bmj.323.7327.1446

125. von Bonin D, Frühwirth M, Heuser P, Moser M. [Effects of speech therapy with poetry on heart rate variability and wellbeing]. Forsch Komplementarmed Klass Naturheilkd. (2001) 8:144-60. doi: 10.1159/000057212

126. Bettermann $H$, von Bonin D, Frühwirth $M$, Cysarz D, Moser $M$. Effects of speech therapy with poetry on heart rate rhythmicity and cardiorespiratory coordination. Int $J$ Cardiol. (2002) 84:77-88. doi: 10.1016/S0167-5273(02)00137-7

127. Cysarz D, von Bonin D, Lackner H, Heusser P, Moser M, Bettermann H. Oscillations of heart rate and respiration synchronize during poetry recitation. Amer J Physiol Heart Circ Physiol. (2004) 287:H579-87. doi: 10.1152/ajpheart.01131.2003

128. Lehrer P, Vaschillo E, Trost Z, France CR. Effects of rhythmical muscle tension at $01 \mathrm{~Hz}$ on cardiovascular resonance and the baroreflex. Biol Psychol. (2009) 81:24-30. doi: 10.1016/j.biopsycho.2009.01.003

129. Vaschillo EG, Vaschillo B, Pandina RJ, Bates ME. Resonances in the cardiovascular system caused by rhythmical muscle tension. Psychophysiology. (2011) 48:927-36. doi: 10.1111/j.1469-8986.2010.01156.x

130. Byrne EA, Porges SW. Frequency-specific amplification of heart rate rhythms using oscillatory tilt. Psychophysiology. (1992) 29:120-6. doi: 10.1111/j.1469-8986.1992.tb02022.x

131. Hamilton LL, Lindan O, Reswick JB. Dynamic effects of sinusoidal tilting on heart rate of healthy and paralyzed persons. J Appl Physiol. (1969) 27:378-84. doi: 10.1152/jappl.1969.27. 3.378

132. Lindqvist A. Noninvasive methods to study autonomic nervous control of circulation. Acta Physiol Scand Suppl. (1990) 588:1-107.

133. Båth E, Lindblad L, Wallin B. Effects of dynamic and static neck suction on muscle nerve sympathetic activity, heart rate and blood pressure in man. $J$ Physiol. (1981) 311:551-64. doi: 10.1113/jphysiol.1981.sp013604

134. Sakakibara M, Hayano J, Oikawa LO, Katsamanis M, Lehrer P. Heart rate variability biofeedback improves cardiorespiratory resting function during sleep. Appl Psychophysiol Biofeedback. (2013) 38:265-71. doi: 10.1007/s10484-013-9232-7

135. Tang H, Qin S, Li W, Chen X, Ulloa L, Zhu Q, et al. P2RX7 in dopaminergic neurons of ventral periaqueductal gray mediates htwp acupuncture- induced 
consciousness in traumatic brain injury. Front Cell Neurosci. (2020) 14:598198. doi: 10.3389/fncel.2020.598198

136. Shaffer F, Meehan ZM. A practical guide to resonance frequency assessment for heart rate variability biofeedback. Front Neurosci. (2020) 14:570400. doi: 10.3389/fnins.2020.570400

137. Shaffer F, Meehan ZM. Corrigendum: A practical guide to resonance frequency assessment for heart rate variability biofeedback. Front Neurosci. (2020) 14:627512. doi: 10.3389/fnins.2020.627512

138. Gevirtz R. The promise of HRV biofeedback: Some preliminary results and speculations. Biofeedback. (2003) 31:18-9. Available online at: https:// www.proquest.com/openview/695b93e0825f395bc5ad1eb0d352e550/1? pqorigsite $=$ gscholar\&cbl $=506344$

139. Gevirtz R. The promise of heart rate variability biofeedback: evidence-based applications. Biofeedback. (2013) 41:110-20. doi: 10.5298/1081-5937-41.3.01

140. Thayer JF, Lane RD. Claude Bernard and the heart-brain connection: further elaboration of a model of neurovisceral integration. Neurosci Biobehav Rev. (2009) 33:81-8. doi: 10.1016/j.neubiorev.2008.08.004

141. Huang C, Gevirtz R, Onton J, Criado JR. Investigation of vagal afferent functioning using the heartbeat event related potential. Int J Psychophysiol. (2018) 131:113-23. doi: 10.1016/j.ijpsycho.2017.06.007

142. Lehrer PM, Vaschillo E, Vaschillo B, Lu SE, Eckberg DL, Edelberg R, et al. Heart rate variability biofeedback increases baroreflex gain and peak expiratory flow. Psychosom Med. (2003) 65:796-805. doi: 10.1097/01.PSY.0000089200.81962.19

143. Vaschillo EG, Vaschillo B, Buckman JF, Pandina RJ, Bates ME, editors. The investigation and clinical significance of resonance in the heart rate and vascular tone baroreflexes. In: Fred A, Filipe J, Gamboa H, editors. Biomedical Engineering Systems and Technologies. BIOSTEC 2010. Communications in Computer and Information Science. Vol. 127. Berlin; Heidelberg: Springer (2010). p. 224-37. doi: 10.1007/978-3-642-18472-7_18

144. Lane R, Reiman E, Ahern G, Thayer J. 21. Activity in medial prefrontal cortex correlates with vagal component of heart rate variability during emotion. Brain Cogn. (2001) 47:97-100. Availble online at: https://arizona. pure.elsevier.com/en/publications/21-activity-in-medial-prefrontal-cortexcorrelates-with-vagalcom

145. MacKinnon S, Gevirtz R, McCraty R, Brown M. Utilizing heartbeat evoked potentials to identify cardiac regulation of vagal afferents during emotion and resonant breathing. Appl Psychophysiol Biofeedback. (2013) 38:24155. doi: 10.1007/s10484-013-9226-5

146. Schandry R, Montoya P. Event-related brain potentials and the processing of cardiac activity. Biol Psychol. (1996) 42:7585. doi: 10.1016/0301-0511(95)05147-3

147. Wölk C. Velden M. Detection variability within the cardiac cycle: toward a revision of the "baroreceptor hypothesis" J Psychophysiol. (1987) 1:61-5.

148. Patron E, Mennella R, Messerotti Benvenuti S, Thayer JF. The frontal cortex is a heart-brake: reduction in delta oscillations is associated with heart rate deceleration. Neuroimage. (2019) 188:403-10. doi: 10.1016/j.neuroimage.2018.12.035

149. Bernard C. Lecture on the Physiology of the Heart and its Connections with the Brain, Delivered at the Sorbonne, the 27th March, 1865. Tr. By J.S. Morel, Savannah, Purse. (1867) (Cited by Thayer \& Lane 2009, p. 81).

150. Darwin C. The Expression of the Emotions in Man and Animals. Chicago, IL: University of Chicago Press (2015). p. 69. Originally published 1872. doi: 10.1037/10001-000

151. Porges SW, Dana DA. Clinical Applications of the Polyvagal Theory: The Emergence of Polyvagal-Informed Therapies (Norton Series on Interpersonal Neurobiology). New York, NY: WW Norton \& Company (2018).

152. Porges SW, Doussard-Roosevelt JA, Maiti AK. Vagal tone and the physiological regulation of emotion. Monogr Soc Res Child Dev. (1994) 59:167-86. doi: 10.1111/j.1540-5834.1994.tb01283.x

153. Kim JJ, Parker SL, Doty JR, Cunnington R, Gilbert P, Kirby JN. Neurophysiological and behavioural markers of compassion. Sci Rep. (2020) 10:1-9. doi: 10.1038/s41598-020-63846-3

154. Burg JM, Wolf OT, Michalak J. Mindfulness as self-regulated attention. Swiss J Psychol. (2012) 71:135-9. doi: 10.1024/1421-0185/a000080

155. Mankus AM, Aldao A, Kerns C, Mayville EW, Mennin DS. Mindfulness and heart rate variability in individuals with high and low generalized anxiety symptoms. Behav Res Ther. (2013) 51:386-91. doi: 10.1016/j.brat.2013.03.005
156. Christodoulou G, Salami N, Black DS. The utility of heart rate variability in mindfulness research. Mindfulness. (2020) 11:554-70. doi: 10.1007/s12671-019-01296-3

157. Davies CD, Niles AN, Pittig A, Arch JJ, Craske MG. Physiological and behavioral indices of emotion dysregulation as predictors of outcome from cognitive behavioral therapy and acceptance and commitment therapy for anxiety. J Behav Ther Exp Psychiatry. (2015) 46:35-43. doi: 10.1016/j.jbtep.2014.08.002

158. Beresnevaite M, Benetis R, Taylor GJ, Rašinskiene S, Stankus A, Kinduris S. Impact of a cognitive behavioral intervention on health-related quality of life and general heart rate variability in patients following cardiac surgery: an effectiveness study. Psychosomatics. (2016) 57:605-15. doi: 10.1016/j.psym.2016. 04.004

159. Patel AI. Cardiovascular Benefits of Forgiveness in Women: A Psychophysiological Study. Thesis, The Ohio State University (2013).

160. Czamanski-Cohen J, Galili G, Allen JJB. Examining changes in HRV and emotion following artmaking with three different art materials. J Vis Exp. (2020) 2020:155. doi: 10.3791/60376

161. Bridges LJ, Denham SA, Ganiban JM. Definitional issues in emotional regulation research. Child Dev. (2004) 75:3405. doi: 10.1111/j.1467-8624.2004.00675.x

162. Chernigovskaya N, Vashchillo E, Petrash V, Rusanovskii V. Voluntary control of the heart rate as a method of correcting the functional state in neurosis. Hum Physiol. (1990) 16:105-111.

163. Moss D, Shaffer F. The application of heart rate variability biofeedback to medical and mental health disorders. Biofeedback. (2017) 45:28. doi: 10.5298/1081-5937-45.1.03

164. McCraty R, Atkinson M, Tiller WA, Rein G, Watkins AD. The effects of emotions on short-term power spectrum analysis of heart rate variability. Am J Cardiol. (1995) 76:1089-93. doi: 10.1016/S0002-9149(99)80309-9

165. Mather M, Thayer JF. How heart rate variability affects emotional regulation brain networks. Curr Opin Behav Sci. (2018) 19:98-104. doi: 10.1016/j.cobeha.2017.12.017

166. Lynar E, Cvejic E, Schubert E, Vollmer-Conna U. The joy of heartfelt music: an examination of emotional and physiological responses. Int $J$ Psychophysiol. (2017) 120:118-25. doi: 10.1016/j.ijpsycho.2017.07.012

167. Koelsch S, Jäncke L. Music and the heart. Eur Heart J. (2015) 36:30439. doi: 10.1093/eurheartj/ehv430

168. Iwanaga M, Kobayashi A, Kawasaki C. Heart rate variability with repetitive exposure to music. Biol Psychol. (2005) 70:616. doi: 10.1016/j.biopsycho.2004.11.015

169. da Silva SA, Guida HL, Dos Santos Antonio AM, de Abreu LC, Monteiro $\mathrm{CB}$, Ferreira $\mathrm{C}$, et al. Acute auditory stimulation with different styles of music influences cardiac autonomic regulation in men. Int Cardiovasc Res J. (2014) 8:105-10. doi: 10.1186/1755-7682-7-27

170. Roque AL, Valenti VE, Guida HL, Campos MF, Knap A, Vanderlei LC, et al. The effects of different styles of musical auditory stimulation on cardiac autonomic regulation in healthy women. Noise Health. (2013) 15:2817. doi: 10.4103/1463-1741.113527

171. Perez-Lloret S, Diez J, Domé MN, Delvenne AA, Braidot N, Cardinali DP, et al. Effects of different "relaxing" music styles on the autonomic nervous system. Noise Health. (2014) 16:279-84. doi: 10.4103/1463-1741.140507

172. Uhlig S, Jaschke A, Scherder E, editors. Effects of music on emotional regulation: a systematic literature review. In: The 3rd International Conference on Music \& Emotion. Jyväskylä: University of Jyväskylä, Department of Music (2013).

173. Cook T, Roy AR, Welker KM. Music as an emotional regulation strategy: an examination of genres of music and their roles in emotional regulation. Psychol Music. (2019) 47:144-54. doi: 10.1177/0305735617 734627

174. Thoma MV, Ryf S, Mohiyeddini C, Ehlert U, Nater UM. Emotional regulation through listening to music in everyday situations. Cogn Emot. (2012) 26:550-60. doi: 10.1080/02699931.2011. 595390

175. Fernández-Sotos A, Fernández-Caballero A, Latorre JM. Influence of tempo and rhythmic unit in musical emotional regulation. Front Comput Neurosci. (2016) 10:80. doi: 10.3389/fncom.2016.00080 
176. Moore KS. A systematic review on the neural effects of music on emotional regulation: implications for music therapy practice. J Music Ther. (2013) 50:198-242. doi: 10.1093/jmt/50.3.198

177. Carlson E, Saarikallio S, Toiviainen P, Bogert B, Kliuchko M, Brattico E. Maladaptive and adaptive emotional regulation through music: a behavioral and neuroimaging study of males and females. Front Hum Neurosci. (2015) 9:466. doi: 10.3389/fnhum.2015.00466

178. Koechlin H, Coakley R, Schechter N, Werner C, Kossowsky J. The role of emotional regulation in chronic pain: a systematic literature review. $J$ Psychosom Res. (2018) 107:38-45. doi: 10.1016/j.jpsychores.2018.02.002

179. Agar-Wilson M, Jackson T. Are emotional regulation skills related to adjustment among people with chronic pain, independent of pain coping? Eur J Pain. (2012) 16:105-14. doi: 10.1016/j.ejpain.2011.05.011

180. Lutz J, Gross RT, Vargovich AM. Difficulties in emotional regulation and chronic pain-related disability and opioid misuse. Addict Behav. (2018) 87:200-5. doi: 10.1016/j.addbeh.2018.07.018

181. Aaron RV, Finan PH, Wegener ST, Keefe FJ, Lumley MA. Emotional regulation as a transdiagnostic factor underlying co-occurring chronic pain and problematic opioid use. Am Psychol. (2020) 75:796-810. doi: 10.1037/amp0000678

182. Joseph B, Shimojo G, Li Z, Thompson-Bonilla MdR, Shah R, Kanashiro A, et al. Glucose activates vagal control of hyperglycemia and inflammation in fasted mice. Sci Rep. (2019) 9:1012. doi: 10.1038/s41598-018-36298-z

183. Renna ME, Fresco DM, Mennin DS. Emotional regulation therapy and its potential role in the treatment of chronic stress-related pathology across disorders. Chronic Stress. (2020) 4:2470547020905787. doi: 10.1177/2470547020905787

184. Roy B, Riley C, Sinha R. Emotional regulation moderates the association between chronic stress and cardiovascular disease risk in humans: a cross-sectional study. Stress. (2018) 21:548-55. doi: 10.1080/10253890.2018.1490724

185. Ragen B, Roach A, Chollak C. Chronic stress, regulation of emotion, and functional activity of the brain. In: Fink G, editors. Stress: Concepts, Cognition, Emotion, and Behavior. Burlington, VA: Academic Press (2016). p. 241-9. doi: 10.1016/B978-0-12-800951-2.00029-7

186. Lopez RB, Denny BT, Fagundes CP. Neural mechanisms of emotional regulation and their role in endocrine and immune functioning: a review with implications for treatment of affective disorders. Neurosci Biobehav Rev. (2018) 95:508-14. doi: 10.1016/j.neubiorev.2018.10.019

187. Miller AH, Capuron L, Raison CL. Immunologic influences on emotional regulation. Clin Neurosci Res. (2005) 4:32533. doi: 10.1016/j.cnr.2005.03.010

188. Appleton AA, Buka SL, Loucks EB, Gilman SE, Kubzansky LD. Divergent associations of adaptive and maladaptive emotional regulation strategies with inflammation. Health Psychol. (2013) 32:748-56. doi: 10.1037/a0030068

189. Wilson SJ, Jaremka LM, Fagundes CP, Andridge R, Peng J, Malarkey WB, et al. Shortened sleep fuels inflammatory responses to marital conflict: emotional regulation matters. Psychoneuroendocrinology. (2017) 79:7483. doi: 10.1016/j.psyneuen.2017.02.015

190. Wilson SJ, Bailey BE, Jaremka LM, Fagundes CP, Andridge R, Malarkey WB, et al. When couples' hearts beat together: synchrony in heart rate variability during conflict predicts heightened inflammation throughout the day. Psychoneuroendocrinology. (2018) 93:107-16. doi: 10.1016/j.psyneuen.2018.04.017

191. Nusslock R, Brody GH, Armstrong CC, Carroll AL, Sweet LH $\mathrm{Yu} \mathrm{T}$, et al. Higher peripheral inflammatory signaling associated with lower resting-state functional brain connectivity in emotional regulation and central executive networks. Biol Psychiatry. (2019) 86:153-62. doi: 10.1016/j.biopsych.2019.03.968

192. Gross JJ, Muñoz RF. Emotional regulation and mental health. Clin Psychol. (1995) 2:151. doi: 10.1111/j.1468-2850.1995.tb00036.x

193. Joormann J, Siemer M. Emotion regulation in mood disorders. In: Gross JJ, editor. Handbook of Emotion Regulation. New York, NY: The Guilford Press (2014). p. 413-27.

194. Joormann J, Gotlib IH. Emotional regulation in depression: relation to cognitive inhibition. Cogn Emot. (2010) 24:28198. doi: 10.1080/02699930903407948
195. Ehring T, Tuschen-Caffier B, Schnülle J, Fischer S, Gross JJ. Emotional regulation and vulnerability to depression: spontaneous versus instructed use of emotion suppression and reappraisal. Emotion. (2010) 10:56372. doi: 10.1037/a0019010

196. Campbell-Sills L, Ellard KK, Barlow DH. Emotion regulation in anxiety disorders. In Gross JJ, editor. Handbook of Emotion Regulation. New York, NY: The Guilford Press (2014). p. 393-412.

197. Sakakibara R, Kitahara M. [The relationship between Cognitive Emotional regulation Questionnaire (CERQ) and depression, anxiety: meta-analysis]. Shinrigaku Kenkyu. (2016) 87:179-85. doi: 10.4992/jjpsy.87.15302

198. Kraiss JT, Ten Klooster PM, Moskowitz JT, Bohlmeijer ET. The relationship between emotional regulation and well-being in patients with mental disorders: a meta-analysis. Compr Psychiatry. (2020) 102:152189. doi: 10.1016/j.comppsych.2020.152189

199. Chin T, Rickard NS. Emotional regulation strategy mediates both positive and negative relationships between music uses and well-being. Psychol Music. (2014) 42:692-713. doi: 10.1177/0305735613489916

200. Renna ME, Hoyt MA, Ottaviani C, Mennin DS. An experimental examination of worry and relaxation on cardiovascular, endocrine, and inflammatory processes. Psychoneuroendocrinology. (2020) 122:104870. doi: 10.1016/j.psyneuen.2020.104870

201. Mennin DS, Fresco DM, Ritter M, Heimberg RG, An open trial of emotional regulation therapy for generalized anxiety disorder and cooccurring. Depress Anxiety. (2015) 32:614-23. doi: 10.1002/da.22377

202. Mennin DS, Fresco DM, O’Toole MS, Heimberg RG. A randomized controlled trial of emotional regulation therapy for generalized anxiety disorder with and without co-occurring depression. J Consult Clin Psychol. (2018) 86:268-81. doi: 10.1037/ccp0000289

203. Renna ME, Quintero JM, Soffer A, Pino M, Ader L, Fresco DM, et al. A pilot study of emotional regulation therapy for generalized anxiety and depression: findings from a diverse sample of young adults. Behav Ther. (2018) 49:403-18. doi: 10.1016/j.beth.2017.09.001

204. Archana R, Mukilan R. Beneficial effect of preferential music on exercise induced changes in heart rate variability. J Clin Diagn Res. (2016) 10:CC0911. doi: 10.7860/JCDR/2016/18320.7740

205. Bernardi L, Porta C, Sleight P. Cardiovascular, cerebrovascular, and respiratory changes induced by different types of music in musicians and non-musicians: the importance of silence. Heart. (2006) 92:44552. doi: 10.1136/hrt.2005.064600

206. Cheng T-H, Tsai C-G. Female listeners' autonomic responses to dramatic shifts between loud and soft music/sound passages: a study of heavy metal songs. Front Psychol. (2016) 7:182. doi: 10.3389/fpsyg.2016.00182

207. Lee KS, Jeong HC, Yim JE, Jeon MY. Effects of music therapy on the cardiovascular and autonomic nervous system in stress-induced university students: a randomized controlled trial. J Altern Complement Med. (2016) 22:59-65. doi: 10.1089/acm.2015.0079

208. Peng S-M, Koo M, Yu Z-R. Effects of music and essential oil inhalation on cardiac autonomic balance in healthy individuals. J Altern Complement Med. (2009) 15:53-7. doi: 10.1089/acm.2008.0243

209. Urakawa K, Yokoyama K. Music can enhance exercise-induced sympathetic dominancy assessed by heart rate variability. Tohoku J Exp Med. (2005) 206:213-8. doi: 10.1620/tjem.206.213

210. Zhou P, Sui F, Zhang A, Wang F, Li G, editors. Music therapy on heart rate variability. In: 2010 3rd International Conference on Biomedical Engineering and Informatics. IEEE. Yantai. (2010).

211. Chang H-K, Peng T-C, Wang J-H, Lai H-L. Psychophysiological responses to sedative music in patients awaiting cardiac catheterization examination: a randomized controlled trial. J Cardiovasc Nurs. (2011) 26:E118. doi: 10.1097/JCN.0b013e3181fb711b

212. Chiu HW, Lin L, Kuo M, Chiang H, Hsu C-Y, editors. Using heart rate variability analysis to assess the effect of music therapy on anxiety reduction of patients. In: Computers in Cardiology. IEEE. Thessaloniki Chalkidiki (2003). doi: 10.1109/CIC.2003.1291194

213. Cotoia A, Dibello F, Moscatelli F, Sciusco A, Polito P, Modolo A, et al. Effects of Tibetan music on neuroendocrine and autonomic functions in patients waiting for surgery: a randomized, controlled study. Anesthesiol Res Pract. (2018) 2018:9683780. doi: 10.1155/2018/9683780 
214. Hsu C-C, Chen W-M, Chen S-R, Tseng Y-T, Lin P-C. Effectiveness of music listening in patients with total knee replacement during CPM rehabilitation. Biol Res Nurs. (2016) 18:68-75. doi: 10.1177/1099800415572147

215. Kemper KJ, Danhauer SC. Music as therapy. South Med J. (2005) 98:2828. doi: 10.1097/01.SMJ.0000154773.11986.39

216. Lai HL, Li YM, Lee LH. Effects of music intervention with nursing presence and recorded music on psycho-physiological indices of cancer patient caregivers. J Clin Nurs. (2012). 21:745-56. doi: 10.1111/j.1365-2702.2011.03916.x

217. Lee KC, Chao YH, Yiin JJ, Chiang PY, Chao YF. Effectiveness of different music-playing devices for reducing preoperative anxiety: a clinical control study. Int J Nurs Stud. (2011) 48:1180-7. doi: 10.1016/j.ijnurstu.2011.04.001

218. Lee Y-C, Lei C-Y, Shih Y-S, Zhang W-C, Wang H-M, Tseng C-L, et al., editors. HRV response of vegetative state patient with music therapy. Annu Int Conf IEEE Eng Med Biol Soc. (2011) 2011:1701-4. doi: 10.1109/IEMBS.2011.60 90488

219. Okada K, Kurita A, Takase B, Otsuka T, Kodani E, Kusama Y, et al. Effects of music therapy on autonomic nervous system activity, incidence of heart failure events, and plasma cytokine and catecholamine levels in elderly patients with cerebrovascular disease and dementia. Int Heart J. (2009) 50:95-110. doi: 10.1536/ihj.50.95

220. Santana MDR, Martiniano EC, Monteiro LRL, Valenti VE, Garner DM, Sorpreso ICE, et al. Musical auditory stimulation influences heart rate autonomic responses to endodontic treatment. Evid Based Complement Altern Med. (2017). 2017:4847869. doi: 10.1155/2017/4847869

221. Wang Y, Dong Y, Li Y. Perioperative psychological and music interventions in elderly patients undergoing spinal anesthesia: effect on anxiety, heart rate variability, and postoperative pain. Yonsei Med J. (2014) 55:11015. doi: 10.3349/ymj.2014.55.4.1101

222. White JM. Effects of relaxing music on cardiac autonomic balance and anxiety after acute myocardial infarction. Am J Crit Care. (1999) 8:22030. doi: 10.4037/ajcc1999.8.4.220

223. Hsu CT, Tai HC, Chung JY, Chen JH, Chen WL. Depressed sympathovagal modulation indicates sepsis in patients with suspected infection. Medicine (Baltimore). (2020) 99:e18961. doi: 10.1097/MD.0000000000018961

224. Lee K-C, Chao Y-H, Yiin J-J, Hsieh H-Y, Dai W-J, Chao YF. Evidence that music listening reduces preoperative patients' anxiety. Biol Res Nurs. (2012) 14:78-84. doi: 10.1177/1099800410 396704
225. Koelsch S, Boehlig A, Hohenadel M, Nitsche I, Bauer K, Sack U. The impact of acute stress on hormones and cytokines, and how their recovery is affected by music-evoked positive mood. Sci Rep. (2016) 6:23008. doi: 10.1038/srep23008

226. Hayano J, Mukai S, Sakakibara M, Okada A, Takata K, Fujinami T. Effects of respiratory interval on vagal modulation of heart rate. Am J Physiol. (1994) 267(1 Pt 2):H33-40. doi: 10.1152/ajpheart.1994.267 .1.H33

227. Desborough JP. The stress response to trauma and surgery. Br J Anaesth. (2000) 85:109-17. doi: 10.1093/bja/85.1.109

228. Huber-Lang M, Lambris JD, Ward PA. Innate immune responses to trauma. Nat Immunol. (2018) 19:327-41. doi: 10.1038/s41590-018-0064-8

229. Khan SH, Kitsis M, Golovyan D, Wang S, Chlan LL, Boustani M, et al. Effects of music intervention on inflammatory markers in critically ill and postoperative patients: a systematic review of the literature. Heart Lung. (2018) 47:489-96. doi: 10.1016/j.hrtlng.2018.05.015

230. Ulloa L. The vagus nerve and the nicotinic anti-inflammatory pathway. Nat Rev Drug Discov. (2005) 4:673-84. doi: 10.1038/nrd1797

231. Ulloa L. Electroacupuncture activates neurons to switch off inflammation. Nature. (2021) 598:573-4. doi: 10.1038/d41586-021-02714-0

Conflict of Interest: The authors declare that the research was conducted in the absence of any commercial or financial relationships that could be construed as a potential conflict of interest.

Publisher's Note: All claims expressed in this article are solely those of the authors and do not necessarily represent those of their affiliated organizations, or those of the publisher, the editors and the reviewers. Any product that may be evaluated in this article, or claim that may be made by its manufacturer, is not guaranteed or endorsed by the publisher.

Copyright (c) 2022 Ginsberg, Raghunathan, Bassi and Ulloa. This is an open-access article distributed under the terms of the Creative Commons Attribution License (CC $B Y)$. The use, distribution or reproduction in other forums is permitted, provided the original author(s) and the copyright owner(s) are credited and that the original publication in this journal is cited, in accordance with accepted academic practice. No use, distribution or reproduction is permitted which does not comply with these terms. 